\title{
Exosomes derived from BMSCs regulate pyroptosis by miR-143-3p/MyD88 axis to ameliorate intestinal ischemia-reperfusion injury
}

\section{Zhanhai Wan}

Lanzhou University

Guangru Zhang

Lanzhou University

\section{Yan Zhang}

Lanzhou University

Jipeng Lv

Lanzhou University

\section{Yuan Yuan}

Lanzhou University

\section{Wenwen Guo}

Lanzhou University

\section{Yufang Leng ( $\nabla$ lengyf@lzu.edu.cn )}

Lanzhou University First Affiliated Hospital https://orcid.org/0000-0001-7094-2413

\section{Research Article}

Keywords: bone marrow mesenchymal stem cells, exosomes, pyroptosis, intestinal ischemia-reperfusion injury, miRNA, MyD88/NF-KB/NLRP3 pathway

Posted Date: March 2nd, 2022

DOI: https://doi.org/10.21203/rs.3.rs-1369094/v1

License: (1) (i) This work is licensed under a Creative Commons Attribution 4.0 International License. Read Full License 


\section{Abstract}

Intestinal ischemia-reperfusion (I/R) injury is a condition in which tissue injury is aggravated after ischemia due to recovery of blood supply. Bone marrow mesenchymal stem cell-derived exosome (BMSCexo) showed a positive effect on I/R injury. This study aimed to investigate the effects and possible mechanism of BMSC-exo on intestinal I/R injury based on pyroptosis. BMSC-exos were isolated by supercentrifugation methods. Cell proliferation and apoptosis in the cell model, and intestinal barrier injury in the mouse model were analyzed to explore the effects of BMSC-exo on intestinal I/R, and inflammatory cytokines and pyroptosis-related proteins were analyzed to explore the effects of BMSC-exo on pyroptosis. In addition, miRNA microarray sequencing was used to analyze the differentially expressed miRNA in the regulation of BMSC-exo in intestinal I/R to further elucidate the mechanism. The results showed that BMSC-exo effectively reduced cell apoptosis, alleviated intestinal barrier injury, and regulated the expression of inflammatory cytokines and pyroptosis-related protein, suggesting that BMSC-exo may alleviate intestinal I/R injury by regulating pyroptosis in vitro and in vivo. In addition, the differentially expressed miRNA screened by miRNA microarray sequencing was miR-143-3p. Bioinformatics analysis predicted that there was a binding site between miR-143-3p and MyD88, and dual-luciferase reporter assay confirmed that miR-143-3p could directly target MyD88 and regulate its expression. Further studies verified that miR-143-3p may regulate pyroptosis by regulating NLRP3 through the TLR4/MyD88/NF-KB pathway and participate in BMSC-exo to alleviat intestinal I/R. In conclusion, this study found that BMSCexo alleviated intestinal I/R by regulating pyroptosis through the miR-143-3p/MyD88/NF-KB/NLRP3 axis.

\section{Introduction}

Ischemia-reperfusion ( $/ / R)$ injury is a common pathophysiological process in many clinical diseases, which is caused by the interruption of blood supply to tissues or organs due to the reduction and subsequent recovery of blood supply to ischemic sites, and reperfusion can not only fail to restore the structure and function of original tissues or organs, but also aggravate the injury[1-2]. The gastrointestinal tract is highly sensitive to I/R injury, and substantial damage to local mucosal tissues may occur even in the case of transient ischemia[3]. Intestinal I/R injury is caused by various pathogenic factors, the pathogenesis is complex, and the mortality is increasing year by year[4]. At present, there is still lack of effective therapeutic methods and drugs in the clinics.

In recent years, stem cell therapy technology has developed rapidly. Studies have shown that mesenchymal stem cells (MSCs) have beneficial effects on inflammation response, immune regulation, tissue perfusion improvement, and intestinal mucosal recovery in intestinal I/R injury[5]. The therapeutic effect of MSCs is mainly through the paracrine mechanism[6]. During I/R injury, the damaged intestine releases pro-inflammatory signals that activate MSCs to release beneficial factors that act in a paracrine manner to treat intestinal I/R injury[7]. Exosomes, as important components in the paracrine manner of stem cells, mediate the communication between cells, and affect the state of recipient cells[8]. Bone marrow MSCs (BMSCs) have been shown to alleviate multiple organ dysfunction syndromes, such as acute lung injury caused by intestinal I/R injury, through exosomes[9], suggesting that the exosomes 
derived from BMSCs (BMSC-exo) may be a promising alternative therapy for intestinal I/R injury. However, its mechanism remains to be further studied.

Pyroptosis is GSDMD-mediated programmed cell death, which is an important mechanism of inflammatory body effect and controls the secretion of cytokines dependent on inflammasome[10]. Inflammation induced by its excessive activation can cause serious tissue and organ damage[11]. Studies have shown that pyroptosis is closely related to many diseases. In recent years, it has been found that pyroptosis and its related proteins are widely present in the process of $I / R$ injury[12]. The activation of NLRP3 inflammasome can recruit and activate the caspase-1 to induce pyroptosis[13], and inhibition of pyroptosis can alleviate I/R injury of various vital organs[14-15]. In addition, studies have shown that BMSC-exo could attenuate pyroptosis induced by oxygen-glucose deprivation/reoxygenation[16], and also attenuate cerebral I/R injury-induced neuroinflammation and pyroptosis[17]. However, the effects and further mechanism of BMSC-exo on pyroptosis in intestinal I/R injury remain unclear.

In this study, the effects and the possible mechanism of BMSC-exo on intestinal I/R injury were investigated in vitro and in vivo based on pyroptosis. And the further mechanism was explored using miRNA microarray sequencing. Our study confirmed that BMSC-exo regulated pyroptosis to alleviate intestinal I/R injury through the miR-143-3p/MyD88/NF-kB/NLRP3 pathway, which may lay a solid theoretical foundation for the application of BMSC-exo in intestinal I/R injury.

\section{Material And Methods}

\section{Animals}

Eighty specific-pathogen-free male C57BL/6 mice aged 8-week and weighing 18-22 g were purchased from Chengdu Dossy Experimental Animals Co., Ltd. (license number: SCXK (Sichuan) 2020-030). Mice were kept at a room temperature of $25^{\circ} \mathrm{C}$, with a humidity of $50-60 \%$, with good ventilation, under a 12-h light/dark cycle and had free access to food and water. The present study was performed in strict accordance with the Guide for the Care and Use of Laboratory Animals of the National Institutes of Health. All experimental protocols of the present study were approved by the First Hospital of Lanzhou University (No. LDYYLL2022-27) and all efforts were made to minimize animal suffering and reduce the number of animals used.

\section{Isolation and culture of BMSCs}

Twenty C57BL/ 6 mice were sacrificed by cervical dislocation, and bone marrow of femur and tibia were extracted. The collected bone marrow fluid was centrifuged at $450 \times \mathrm{g}$ at room temperature for $20 \mathrm{~min}$, and then cultured on Dulbecco's modified eagle medium (DMEM, Hyclone, USA) containing $20 \%$ fetal bovine serum (FBS, Gibco, USA) at $37^{\circ} \mathrm{C}$ with $5 \% \mathrm{CO}_{2}$. After $24 \mathrm{~h}$, the suspension cells were removed, and then the medium was replaced every 3 days until the cells reached about $80 \%$ confluence. After being cultured to the third generation, the morphology of BMSCs was observed under a light microscope 
(Olympus, Tokyo, Japan), the differentiation of BMSCs was induced to identify the osteogenic or adipogenic ability, and the surface markers were measured by flow cytometry.

\section{Osteogenic and adipogenic induction of BMSCs}

BMSCs at the third passage were inoculated into 6-well plates at a density of $1 \times 10^{5}$ cells per well. After cells reached $80 \%$ confluence, the original medium was replaced by osteogenic or adipogenic induction medium (osteogenic induction medium: DMEM including 10\% FBS, $5 \mu \mathrm{g} / \mathrm{mL}$ insulin, $0.1 \mu \mathrm{M}$ dexamethasone, $0.2 \mathrm{mM}$ vitamin $\mathrm{C}$ and $10 \mathrm{mM} \beta$-glycerophosphate; adipogenic induction medium: DMEM including $10 \% \mathrm{FBS}, 10 \mu \mathrm{g} / \mathrm{mL}$ insulin, $1 \mu \mathrm{M}$ dexamethasone, $0.5 \mathrm{mM}$ IBMX, $0.1 \mathrm{mM}$ indomethacin) for two weeks to induce the differentiation of BMSCs into osteoblast or adipocyte. The medium was replaced every 3 days. The osteogenic and adipogenic differentiation of BMSCs were assessed by alizarin red $\mathrm{S}$ staining and oil red $\mathrm{O}$ staining, respectively. Briefly, after BMSCs were rinsed with PBS (Solarbio, China) and immobilized with $4 \%$ paraformaldehyde (Solarbio, China) at room temperature for $20 \mathrm{~min}$, cells were stained with alizarin red S (Sigma-Aldrich, USA) at room temperature for $5 \mathrm{~min}$ or oil red $\mathrm{O}$ (Sigma-Aldrich, USA) at room temperature for $30 \mathrm{~min}$. Then the dye was washed away with PBS, and hematoxylin was dyed for $1 \mathrm{~min}$, and then washed with PBS. The images were analyzed by an inverted microscope (Olympus, Tokyo, Japan).

\section{Flow cytometry assays}

The surface markers of BMSCs were measured by flow cytometry, about $1 \times 10^{6}$ third-generation BMSCs were collected and resuspended with $100 \mu \mathrm{L}$ PBS. Then, $5 \mu \mathrm{L}$ antibodies including CD29 (cat. no. 102222), CD34 (cat. no. 343527), CD45 (cat. no. 157212), and CD105 (cat. no. 323205; 1:20; all BioLegend, Inc., USA) were appended into the mixtures and incubated at $4{ }^{\circ} \mathrm{C}$ for $30 \mathrm{~min}$. After incubation, the cells were rinsed with PBS, centrifuged at $1000 \mathrm{rpm}$ for $5 \mathrm{~min}$ at room temperature, and the supernatant was discarded. The cells were suspended by PBS and analyzed by a FACSCalibur flow cytometer (BD Biosciences, USA).

\section{BMSCs transfection}

BMSCs at the third generation were inoculated into 6-well plates at a density of $1 \times 10^{5}$ cells per well and cultured at $37^{\circ} \mathrm{C}$ overnight. Then $50 \mathrm{pmol} / \mathrm{mL}$ miR-143-3p mimic, negative control mimic (NC mimic), miR-143-3p inhibitor and NC inhibitor (Guangzhou RiboBio Co., Ltd., Guangdong, China) were transfected into BMSCs by Lipofectamine 2000 (Invitrogen, Thermo Fisher Scientific, USA) based on the operating instructions. Follow-up experiments were performed $48 \mathrm{~h}$ after transfection.

\section{Extraction and identification of exosomes}

The exosomes of BMSCs or transfected BMSCs (BMSC-exo, miR-143-3p mimic-exo, NC mimic-exo, miR143-3p inhibitor-exo and NC inhibitor-exo) were extracted by super-centrifugation methods. Briefly, BMSCs or transfected BMSCs were cultured for $24 \mathrm{~h}$ in culture medium without exosomes, and $50 \mathrm{~mL}$ supernatant was collected, centrifuged at $4^{\circ} \mathrm{C}$ for $30 \mathrm{~min}$ at $2000 \times \mathrm{g}$, and then centrifuged again at $10000 \times \mathrm{g}$ at $4^{\circ} \mathrm{C}$ for $45 \mathrm{~min}$ to remove large cell fragments and microbubbles. Then, the supernatant was 
filtered through a $0.45 \mu \mathrm{m}$ filter and the filtrate was centrifuged at $4^{\circ} \mathrm{C}$ at $100000 \times \mathrm{g}$ for $70 \mathrm{~min}$. The precipitate was dissolved in $10 \mathrm{~mL}$ PBS, centrifuged at $100000 \times \mathrm{g}$ at $4^{\circ} \mathrm{C}$ for $70 \mathrm{~min}$ again, and resuspended with $100 \mu \mathrm{L}$ PBS. Then the exosomes were stained with $10 \mu \mathrm{L}$ PKH-67 fluorescence dye working solution (cat. no. UR52303, Umibio; China) at room temperature for 10 min away from light. And the morphology of exosomes was observed by transmission electron microscopy (TEM, HT-7700, Hitachi; Japan), the particle size of exosomes was determined by nanoparticle tracking analysis (NTA, N30E, NanoFCM; China), and the markers of CD9, CD63 and TSG101 were determined by western blot analysis.

\section{Cell experiment}

IEC-6 cells were obtained from American Type Culture Collection (Rockville, MD, USA), and cultured in DMEM (Hyclone, USA) supplied with 10\% FBS (Gibco, USA) and 1\% streptomycin and penicillin (Sigma Aldrich, USA) at $37^{\circ} \mathrm{C}$ with $5 \% \mathrm{CO}_{2}$. The cell experiments were divided into two parts. The first part was divided into the control group, the oxygen-glucose deprivation/reoxygenation (OGD/R) group, and the BMSC-exo group. The second part was divided into control group, OGD/R group, WT-exo group, NC mimicexo group, miR-143-3p mimic-exo group, NC inhibitor-exo group, and miR-143-3p inhibitor-exo group. OGD/R cell model was established by incubating the cells in an incubator filled with $1 \% \mathrm{O}_{2}, 5 \% \mathrm{CO}_{2}$ and $94 \% \mathrm{~N}_{2}$ for $24 \mathrm{~h}$, and then oxygenated for $6 \mathrm{~h}$. After the cell model was established, cells in each group were treated with $5 \mu \mathrm{g} / \mathrm{mL}$ corresponding BMSC-exo for $24 \mathrm{~h}$.

\section{Cell viability assay}

IEC-6 cells were inoculated into 96 -well culture plates at a density of $5 \times 10^{3}$ per well and treated with different methods, the cell viability was detected using a Cell Counting Kit-8 (CCK-8; Beyotime, China) at $37^{\circ} \mathrm{C}$ for $1 \mathrm{~h}$. The absorbance was measured at $450 \mathrm{~nm}$ using a microplate spectrophotometer (cat. no. 1681150; Bio-Rad, USA) and cell viability was calculated.

\section{Cell apoptosis assay}

IEC-6 cells under different treatments were collected and stained with Annexin V-APC and PI (Sigma Aldrich, USA) at room temperature for $20 \mathrm{~min}$ in the dark. The fluorescence of the cells was measured by the FACSCalibur flow cytometer (BD Biosciences, USA).

\section{Animals experimental design}

The animal experiments were also divided into two parts. In the first part, twenty-five male C57BL/6 mice were randomly divided into the control group, l/R model group, BMSC group, BMSC-exo group and BMSCGW4869 group. In the second part, thirty-five mice were randomly divided into the control group, I/R group, WT-exo group, NC mimic-exo group, miR-143-3p mimic-exo group, NC inhibitor-exo group, and miR143-3p inhibitor-exo group, with 5 mice in each group. Except for the control group, the I/R animal model was constructed in each group. Mice were anesthetized by intraperitoneal injection of $50 \mathrm{mg} / \mathrm{kg}$ pentobarbital sodium, the superior mesenteric artery was isolated, clipped for $45 \mathrm{~min}$, the vascular clamp was relaxed, and then continued perfusion for $2 \mathrm{~h}$. In the control group, the superior mesenteric artery was isolated but not clipped. The mice in the BMSC group, BMSC-exo group/WT-exo group, BMSC-GW4869 
group, NC mimic-exo group, miR-143-3p mimic-exo group, NC inhibitor-exo group and miR-143-3p inhibitor-exo group were intraperitoneally injected with $200 \mu \mathrm{L} 10^{7} / \mathrm{mL}$ BMSCs, $50 \mu \mathrm{g} \mathrm{MSC}$-exo, $200 \mu \mathrm{L}$ $10^{7} / \mathrm{mL}$ GW4869 pretreated BMSC, $50 \mu \mathrm{g} \mathrm{NC}$ mimic-exo, $50 \mu \mathrm{g}$ miR-143-3p mimic-exo, $50 \mu \mathrm{g}$ NC inhibitor or $50 \mu \mathrm{g}$ miR-143-3p inhibitor-exo, respectively. The control and I/R group were intraperitoneally injected with $200 \mu \mathrm{L}$ PBS. After $24 \mathrm{~h}$, the mice were sacrificed by cervical dislocation, and intestinal tissues and blood were collected.

\section{Hematoxylin-eosin staining}

The intestinal tissues were fixed in $4 \%$ paraformaldehyde at room temperature for $24 \mathrm{~h}$, paraffinembedded and sliced. The slices were dewaxed with xylene and ethanol gradient, and then stained with hematoxylin and eosin staining for pathological studies. Each section of colonic tissue was evaluated by light microscopy (Olympus, Tokyo, Japan) using Chiu's score, ranging from 1 to 5 , with the following criteria[18]: 0 represents normal mucosa; 1 represents the occurrence of Gruenhagen's gap under the intestinal mucosa epithelium in the central axis of the villi; 2 represents the elevation of the intestinal mucosa epithelium from the membrane propria and the expansion of the subepithelial gap; 3 represents the elevation of large tracts of intestinal mucosa epithelium, the villi fall to the sides, and part of the villi fall off; 4 represents shedding of villi and propria, dilatation of capillaries, and increase of cell composition of propria; 5 represents decay or digestion of propria, bleeding or ulceration. The score was expressed as the average of the evaluation of five animals per group.

\section{Immunofluorescent staining}

The intestinal tissue slices were antigen repaired and blocked by bovine serum albumin for $1 \mathrm{~h}$. Then the primary anti-ASC (1:100, cat. no. sc-514414) or anti-Caspase-1 (1:100, cat. no. sc-392736) antibodies were co-incubated with anti-NLRP3 (1:100; cat. no. \# PA5-88709, Invitrogen, USA) at $4{ }^{\circ} \mathrm{C}$ overnight, and the fluorescent secondary antibodies Alexa Fluor 488-labeled Goat Anti-Mouse $\lg G(H+L)(1: 500$; cat. no. A0428) and Alexa Fluor 647-labeled Goat Anti-Rabbit IgG(H+L) (1:500; cat. no. A0468; Beyotime Biotechnology, China) were used to incubate at room temperature and away from light for $1 \mathrm{~h}$. Finally, the DAPI (Sigma, USA) was used for re-staining the nucleus for $5 \mathrm{~min}$ and observed under a microscope (Olympus, Tokyo, Japan).

\section{Western blot analysis}

The expression of marker proteins CD9, CD63 and TSG101 in BMSC-exo, and Claudin 1, Occludin, Bax, Bcl-2, NLRP3, ASC, Caspase-1, cleaved-N-GSDMD, TLR4, p-NF-KB, NF-KB and MyD88 in IEC-6 cells or intestinal tissues of mice were detected by western blot analysis. Total proteins were isolated using RIPA lysis buffer (Beyotime, China), and detected with the BCA protein assay kit (Beyotime, China). Protein samples were separated by $10 \%$ SDS-PAGE and transferred onto a PVDF membrane (EMD Millipore, Boston, USA). The membranes were blocked for $1 \mathrm{~h}$ at room temperature in TBST containing 5\% skimmed milk, and then incubated with primary antibodies (CD9, 1:2000, cat. no. ab263019; CD63, 1:2000, cat. no. ab134045; TSG101, 1:2000, cat. no. ab125011; Claudin 1, 1:2000, cat. no. ab180158; Occludin, 1:1000, cat. no. ab216327; Bax, 1:2000, cat. no. ab32503; Bcl-2, 1:1000, cat. no. ab194583; 
MyD88, 1:1000; cat. no. ab219413; obtained from Abcam, UK. cleaved-N-GSDMD, 1:1000, cat. no. \#10137; phospho-NF-KB, 1:1000, cat. no. \#3033; NF-kB, 1:1000, cat. no. \#8242; obtained from Cell Signaling Technology, USA. TLR4, 1:1000, cat. no. sc-293072; ASC, 1:1000, cat. no. sc-514414; Caspase-1, 1:1000, cat. no. sc-392736; obtained from Santa Cruz Biotechnology, USA. NLRP3, 1:1000; cat. no. \# PA588709; obtained from Invitrogen, USA) overnight at $4^{\circ} \mathrm{C}$. After being rinsed with TBST three times, the membrane was incubated with the HRP-conjugated secondary antibody (ab205719 or ab205718, 1: $10000, A b c a m, U K)$ for $1 \mathrm{~h}$ at room temperature. The protein bands were visualized and analyzed with the enhanced chemiluminescence detection kit (Bio-Rad, USA) and a ChemiDoc ${ }^{\mathrm{TM}}$ MP imaging system (BioRad, USA).

\section{Enzyme-linked immunosorbent assay}

The expression levels of TNF- $\alpha$, IL-1 $\beta$, IL-18, IL-10, TGF- $\beta 1$, DAO and MPO in IEC- 6 cells or serum of mice were examined by ELISA. The blood was collected and centrifuged at $1500 \times \mathrm{g}$ for $15 \mathrm{~min}$, and serum was collected. The ELISA detection procedure was carried out in strict accordance with the product instructions (Shanghai KALANG, China) with the double antibody sandwich enzyme-linked immunosorbent method.

\section{Dual-luciferase reporter assay}

MyD88 wild-type and its corresponding mutant were built into pGL3-basic luciferase vector $(2 \mu \mathrm{g} / \mathrm{mL}$; Shanghai GenePharma Co., Ltd., China) to generate the luciferase reporter plasmids. Cells at the third passage were inoculated into 24 -well plates at a density of $4 \times 10^{4}$ cells per well and cultured at $37^{\circ} \mathrm{C}$. When reached about $70 \%$ confluence, cells were co-transfected with luciferase reporter plasmids, miR143-3p mimic or NC mimic by using Lipofectamine 2000 (Invitrogen; Thermo Fisher Scientific, USA) according to the operating instructions. Luciferase activity was examined with a dual-luciferase reporter assay kit (cat. no. E1910; Promega Corporation, Madison, USA) following transfection for $24 \mathrm{~h}$.

\section{Real-time quantitative polymerase chain reaction (RT-PCR)}

The total RNA from cells or mice was prepared using an RNA Sample Total RAN kit (Tiangen Biotech Co., Ltd.), and cDNA was obtained using the PrimeScript ${ }^{\text {TM }}$ RT reagent kit (Takara, China). RT-PCR was carried out on an Applied Biosystems 7500 Real-Time PCR system (Applied Biosystems; Thermo Fisher Scientific; UAS) through the SYBR Premix Ex Taq II (Takara, China). For the quantification of miRNA level, RT-PCR was executed by the Bulge-Loop ${ }^{\text {TM }}$ miRNA RT-qPCR Primer and Bulge-Loop ${ }^{\text {TM }}$ miRNA RT-qPCR Starter kit (cat. no. C10211-1; Guangzhou RiboBio Co., Ltd., China). The relative gene expression levels were determined using the comparative $2^{-\triangle \Delta C q}$ method with actin or $\mathrm{U} 6$ small nuclear RNA genes as the endogenous controls. The primer sequences were as follows: MyD88 (Forward primer: 5'-

TCGACGCCTTCATCTGCTAC-3', Reverse primer: 5'- CCATGCGACGACACCTTTTC-3'); Actin (Forward primer: 5'- GAAGATCAAGATCATTGCTCC-3', Reverse primer: 5'-TACTCCTGCTTGCTGATCCA-3'), miR-1433p (Forward primer: 5'- GTGAGATGAAGCACTGTAGC-3', Reverse primer: 5'- GTGAGATGAAGCACTGTAGC3'), and U6 (Forward primer: 5'- CTGGTACATCAGCACCTCAC-3', Reverse primer: 5'-

AGAAACAGTCCAGCCCATAC-3'). 


\section{miRNA microarray sequencing}

Intestinal tissues of the control, model and BMSC-exo group were collected to extract total RNA, and the concentration and purity of total RNA were examined by agarose electrophoresis and Agilent 2100 Bioanalyzer. The miRNA libraries were constructed using Illumina TruSeg Small RNA Sample Prep Kit, and sequencing was performed with an Hiseq Single-End of Illumina platform (USA).

\section{Statistical analysis}

All data were analyzed using SPSS 22.0 (IBM, USA) statistical analysis software, and shown as mean \pm standard deviation (SD). Differences between two groups were determined by unpaired Student's t-test, and differences among multiple groups were analyzed using one-way analysis of variance (ANOVA) with Dunnett's post hoc test. $P<0.05$ was considered to indicate a statistically significant difference.

\section{Results}

\section{Extraction and identification of BMSCs and BMSC-exo}

BMSCs were isolated from mouse bone marrow and cultured by whole cell adherent method, the third generation of cells was fusiform, fibrous and irregular shape (Fig. 1A). And the results of osteogenic and adipogenic differentiation showed that BMSCs from mice had multidirectional differentiation potentials for osteogenesis (Fig. 1B) and adipogenesis (Fig. 1C). In addition, the markers of BMSCs were examined and confirmed that the CD29 and CD105 were positive and CD34 and CD45 were negative in BMSCs we isolated (Fig. 1D). These results confirmed that the isolated cells were identified as BMSCs. BMSC-exo were further obtained by super-centrifugation, and the morphology of exosomes was shown in Fig. 1E. The concentration of exosome particles was $7.86 \times 10^{10}$ particles $/ \mathrm{mL}$, and the average particle size was $80.53 \mathrm{~nm}$ (Fig. 1F). Protein quantification results showed that the concentration of exosome solution was $2.50 \mu \mathrm{g} / \mu \mathrm{L}$, indicating that $1 \mu \mathrm{g}$ exosome contains $3.144 \times 10^{7}$ exosome particles. In addition, exosome surface markers TSG101, CD63 and CD9 were positively expressed in BMSC-exo (Fig. 1G), confirming the successful isolation of exosomes from BMSCs.

\section{Effects of BMSC-exo on OGD/R cell model}

The effects of BMSC-exo on intestinal I/R injury were analyzed based on the OGD/R cell model. Fluorescence images of PKH-67-labeled BMSC-exo co-cultured with IEC-6 cells at different times were shown in Fig. 4A. Cell viability (Fig. 4B) and apoptosis (Fig. 4C and D) analysis showed that compared with the control group, the cell viability was significantly reduced and apoptosis was significantly increased in the OGD/R model group $(\mathrm{P}<0.001)$, and BMSC-exo treatment significantly increased the cell viability and reduced cell apoptosis of the OGD/R group $(P<0.01)$. ELISA assay of inflammatory cytokines showed that the expressions of IL-1 $\beta$, IL-18 and TNF- $\alpha$ in the OGD/R group were significantly increased compared with the control group, while BMSC-exo significantly reduced the expressions of these indicators in the OGD/R group $(\mathrm{P}<0.05$, Fig. 4E). In addition, western blot analysis (Fig. 4F and 4G) 
showed that compared with the control group, the expressions of NLRP3, ASC, Caspase-1 and cleaved-NGSDMD in the OGD/R group were significantly increased, while BMSC-exo significantly reverse the change of the protein expression $(P<0.001)$. These results suggested that BMSC-exo could alleviate the inflammatory response of $\mathrm{OGD} / \mathrm{R}$ cells, reduce cell pyroptosis, and play a positive role in intestinal I/R injury.

\section{Effects of BMSC-exo on I/R model mice}

To further confirm the effects of BMSC-exo on intestinal I/R injury, follow-up experiments were conducted in vivo using an I/R mouse model. Histopathological results (Fig. 3A) showed that in the l/R model group, cells in the mucosal layer were necrotic and nucleolysis, inflammatory cells, mainly lymphocytes and plasma cells, were infiltrated in the necrotic area. Bleeding, overflow of red blood cells, hyperemia and expansion of submucosal vessels, muscle layer necrotic and eosinophilic enhancement were also observed. And BMSC, BMSC-exo and BMSC-GW4869 all improved intestinal injury to a certain extent, among which the effect of BMSC-exo was better than that of BMSC, and the effect of BMSC-G W4869 was the worst, as shown by Chiu's score $(P<0.05$, Fig. $3 B)$. The activity of DAO in the blood can reflect the damage and repair of intestinal barrier mucosa, the present study found that the expressions of DAO in the serum of I/R mice were significantly increased, while BMSCs and BMSC-exo significantly reversed the increase of DAO, and GW4869 inhibited the effects of BMSC-exo $(P<0.05$, Fig. 3C). In addition, western blot analysis also showed that compared with the control group, the expressions of Claudin 1 , Occludin and Bcl-2 were significantly decreased, while the expression of Bax was significantly increased in the I/R group. BMSCs and BMSC-exo significantly reversed the decrease of Claudin 1, Occludin and $\mathrm{Bcl}-2$ and the increase of Bax in the I/R group, and the regulation of BMSC-exo was significantly better than that of BMSCs and was significantly inhibited by GW4869 $(P<0.01$, Fig. 3D and 3E). These results suggested that BMSC-exo could significantly improve intestinal barrier injury and reduce intestinal cell apoptosis.

In order to further analyze the regulatory mechanism of BMSC-exo on intestinal I/R injury, the expressions of pro-inflammatory cytokines IL-1 $\beta$, IL-18 and TNF-a (Fig. 4A), anti-inflammatory cytokines IL-10 and TGF- $\beta 1$ (Fig. 4B), and MPO (Fig. 4C) were analyzed by ELISA, and the results showed that compared with the control group, the expressions of IL-1 $\beta$, IL-18, TNF- $a$ and MPO in the I/R group were significantly increased, while IL-10 and TGF- $\beta 1$ were significantly decreased $(P<0.01)$. BMSCs and BMSC-exo significantly reversed the changes of these indicates in the I/R group, while GW4869 inhibited the effects of BMSC-exo $(P<0.05)$. In addition, analysis of pyroptosis-associated protein expression showed that compared with the control group, the expressions of NLRP3, ASC, Caspase- 1 and cleaved-N-GSDMD were significantly increased in the I/R group, while BMSCs, BMSC-exo and BMSC-GW4869 significantly reversed the increase of these proteins in the I/R group, and the regulation of BMSC-exo was significantly better than that of BMSCs, and was significantly inhibited by GW4869 $(P<0.05$, Fig. 4D and 4E).

\section{Effects of BMSC-exo on miRNA expression profile in intestinal tissues of I/R mice}


Exosomes generally play a role by targeting target genes through miRNAs. In order to further clarify the regulatory mechanism of BMSC-exo on intestinal I/R injury, miRNA microarray was used to detect the changes of miRNA expression profile in intestinal tissues. Pearson correlation coefficient was used to represent the correlation between miRNA expression levels among samples, as shown in Fig. 5A, the correlation coefficient between each sample was $0.8-1$, showing a strong correlation, which indicated that the experiment was reliable and the sample selection was reasonable. Bidirectional clustering analysis was performed for all miRNAs and samples by Pheatmap software package, and clustering was performed according to the expression level of the same miRNA in different samples and the expression patterns of different miRNAs in the same sample, the distance was calculated by Euclidean method and complete linkage was used for clustering. The result was shown in Fig. 5B. In addition, the principal component analysis (PCA) results showed that the samples in each group were close to each other and had a high similarity. Similarly, the discrete distribution of the three groups of samples indicates that there are certain differences among groups (Fig. 5C). DESeq (Version 1.18.0) was further used to analyze the differential expression of miRNA in each sample, and the differences of conservative miRNAs were screened out according to the expression of multiple difference $\left(\| \log _{2}\right.$ Fold Change| $\left.>1\right)$ and the expression difference significance $(P<0.05)$. The screening results were shown in Fig. 5D. There were 5 up-regulated miRNAs and 13 down-regulated miRNAs between the control and I/R group, 15 up-regulated and 27 down-regulated miRNAs between the control and BMSC-exo group, and 5 up-regulated and 4 down-regulated miRNAs between I/R and BMSC-exo group. The Venn diagram of miRNA differential expression was shown in Fig. 5E, and the overlapping miRNA in the three groups was miR-143-3p, which was significantly reduced in the I/R group compared with the control group, and significantly increased in the BMSC-exo group. Therefore, miR-143-3p was screened out as the key miRNA for our follow-up study according to the results of miRNA microarray, and its expression was verified on the basis of animal and cell models. RT-PCR results showed that the expression of miR-143-3p was significantly decreased in both the I/R animal model and the OGD/R cell model, which was significantly increased by BMSC-exo ( $P$ $<0.05$, Fig. $5 \mathrm{~F}$ and $5 \mathrm{G})$, consistent with the sequencing results.

\section{miR-143-3p directly targeted MyD88 and negatively modulated its expression}

To further clarify the role of miR-143-3p in intestinal I/R injury, bioinformatics analysis was used to analyze the target genes of miR-143-3p. And it revealed that there was a binding site between the $3^{\prime}-U T R$ of MyD88 and miR-143-3p due to the complementary base pairing (Fig. 6A). Dual-luciferase reporter assay showed that the relative luciferase activity of co-transfection of MyD88 (wt) and miR-143-3p mimic was significantly decreased compared with that of co-transfection of MyD88 (wt) and NC mimic ( $\mathrm{P}<$ 0.001), and no statistical differences were observed in the relative luciferase activity of co-transfection of MyD88 (mut) and miR-143-3p mimic or NC mimic (Fig. 6B). Thus, there was a direct binding between miR-143-3p and MyD88. Further analysis of the regulatory relationship between miR-143-3p and MyD88 showed that both relative gene and protein expressions of MyD88 were significantly decreased after miR143-3p mimic treatment compared with NC mimic, while the expressions were significantly increased 
after miR-143-3p inhibitor treatment compared with NC inhibitor ( $P<0.01$, Fig. 6C and 6D). Therefore, these results suggested that miR-143-3p directly targeted MyD88 and negatively modulated its expression.

\section{BMSC-exo regulated pyroptosis in OGD/R cell models through the miR-143-3p-MyD88 axis}

To further clarify the role of miR-143-3p in the regulation of BMSC-exo in intestinal I/R injury, OGD/R model cells were treated with miR-143-3p mimic-exo or miR-143-3p inhibitor-exo, respectively. The mRNA expression of miR-143-3p was detected by RT-PCR, and results showed that the expression of miR-143-3p was significantly increased after miR-143-3p mimic-exo treatment and significantly decreased after miR143-3p inhibitor-exo treatment $(P<0.05$, Fig. 7A). And cell viability of exo-treated cells was significantly increased after miR-143-3p mimic-exo treatment and significantly decreased after miR-143-3p inhibitorexo treatment $(P<0.05$, Fig. 7B). Similarly, the expressions of IL-1 $\beta$, IL-18 and TNF-a in the exo-treated cells were significantly decreased by miR-143-3p mimic-exo, and the expressions of IL-1 $\beta$ and IL-18 were significantly increased by the miR-143-3p inhibitor-exo $(P<0.05$, Fig. 6C). For pyroptosis, miR-143-3p mimic-exo significantly decreased the pyroptosis rate of cells and miR-143-3p inhibitor-exo showed the opposite effect on the pyroptosis ( $\mathrm{P}<0.001$, Fig. 7D). In addition, miR-143-3p mimic-exo significantly decreased the expression of TLR4, MyD88, p-NF-KB/NF-KB, NLRP3, ASC, caspase-1 and cleaved-NGSDMD compared with the NC mimic-exo group, and miR-143-3p inhibitor-exo significantly increased these protein expressions compared with the NC inhibitor-exo group $(P<0.001$, Fig. 7E). These results suggested that BMSC-exo could regulate pyroptosis in the OGD/R cell model through miR-1433p/MyD88/NF-KB/NLRP3 pathway.

\section{BMSC-exo alleviated intestinal I/R injury in I/R mice model through the miR-143-3p-MyD88 axis}

The effects of miR-143-3p on intestinal I/R injury were further verified in the mice model. As shown in Fig. 8A and 8B, the HE results showed that miR-143-3p mimic-exo alleviated histopathological injury of I/R, and the effect was better than NC mimic-exo, while the effect of miR-143-3p inhibitor-exo was not as good as NC inhibitor-exo. ASC, Caspase-1 and NLRP3 fluorescence double staining results showed that the expressions of ASC and caspase-1 in the miR-143-3p mimic-exo group were significantly decreased compared with the NC mimic-exo group, and miR-143-3p inhibitor-exo significantly increased their expressions compared with the NC inhibitor-exo group ( $\mathrm{P}<0.05$, Fig. 8C-8F), suggesting miR-143-3p was involved in the regulation of pyroptosis by BMSC-exo. Further research showed that the expressions of IL$1 \beta, I L-18$ and TNF- $a$ were significantly decreased by miR-143-3p mimic-exo, while these expressions were significantly increased by miR-143-3p inhibitor-exo $(P<0.05$, Fig. 9A). And miR-143-3p mimic-exo could significantly decrease the expression of TLR4, MyD88, p-NF-KB/NF-KB, NLRP3, ASC, caspase-1 and cleaved-N-GSDMD in I/R compared with NC mimic-exo, and the regulation effects of miR-143-3p inhibitorexo were inferior to NC inhibitor-exo $(P<0.01$, Fig. 9B and 9C). 


\section{Discussion}

Intestinal I/R injury is a common tissue and organ injury, which can lead to the injury of distant organs, with high morbidity and mortality[19]. MSCs have proven to be a powerful tool for repairing damaged organs through paracrine action[5]. Paracrine can explain the immunomodulatory, anti-inflammatory, tissue repair, blood vessel regeneration and antioxidant activity of MSCs[20]. Exosomes originate from a variety of cell types, and releases cell contents into other cells through endocytosis, ligand-receptor and plasma membrane fusion, mediating intercellular communication, and influencing the physiology and pathology of recipient cells[21]. Compared with cellular therapy, MSCs-exo is a promising cell-free alternative with reduced immunogenicity and carcinogenic potential and excellent biocompatibility to deliver its bioactive constituents[22]. Studies have shown that BMSC-exo plays a positive role in various I/R injuries[23-24]. This study confirmed that BMSC-exo isolated from mouse bone marrow can promote cell proliferation and reduce apoptosis in OGD/R cell model in vitro, and reduce intestinal mucosal injury and intestinal cell apoptosis in I/R animal model in vivo, showing positive effects on intestinal I/R injury. In addition, BMSC-exo can significantly regulate inflammatory response and pyroptosis in intestinal I/R injury both in vitro and in vivo.

Pyroptosis, also known as inflammatory cell necrosis, is a caspase-dependent type of programmed cell death[25]. Under the stimulation of pathogens or inflammatory factors, pattern recognition receptors (PRRs) promote the combination of apoptosis-associated speck-like protein containing (ASC) and procaspase- 1 to form inflammasome, thereby activating caspase-1[26]. Activated caspase-1 cleaved GSDMD to form a peptide containing N-terminal active domain on the one hand, and cleaved the precursors of IL-1 $\beta$ and IL-18 on the other hand, resulting in pyroptosis[13]. Excessive pyroptosis in some chronic diseases is harmful to the host and may aggravate the diseases[27]. Studies have shown that pyroptosis has potential as a therapeutic target for many human diseases, and blocking pyroptosis with compounds can treat inflammatory diseases[28]. In addition, the inflammatory response and related proteins of pyroptosis are closely related to the pathogenesis of I/R injury[29-30], suggesting that pyroptosis may play an important role in the treatment of intestinal I/R injury. In this study, we found that BMSC-exo significantly reduced the expression of IL-1 $\beta$ and IL-18, and decreased the protein expression of NLRP3, ASC, caspase-1 and cleaved-N-GSDMD in intestinal I/R injury in vitro and in vivo, confirming that BMSC-exo may alleviate intestinal I/R injury by pyroptosis.

Exosomes, as extracellular vesicles with double lipid structure, carry RNAs (mRNA, miRNA, IncRNA and rRNA), lipids and proteins, etc[31]. MiRNAs are a class of small coding RNAs that are evolutionarily conserved and exhibit important physiological effects through post-transcriptional regulation of their messenger RNA targets[32]. Studies have shown that miRNAs are involved in a variety of $I / R$ injuries and may be key targets for I/R treatment[33]. In this study, the differentially expressed miR-143-3p, which was significantly decreased in the I/R model group and significantly upregulated in the BMSC-exo group, was identified by miRNA microarray sequencing. The validation results in vitro and in vivo were consistent with the sequencing results. Previous study has confirmed that MSC-derived exosomal miR-143-3p inhibits myocardial I/R injury by regulating autophagy[34]. This study showed that miR-143-3p can 
regulate pyroptosis in vitro and in vivo to alleviate intestinal I/R injury. And bioinformatics analysis showed that there was a binding site between miR-143-3p and myeloid differentiation primary response gene 88 (MyD88). Myd88, as a core player in innate immune signaling, is a key connector molecule of the toll-like receptor (TLR) signaling pathway, and plays an important role in the transmission of upstream information and the occurrence and development of diseases[35]. This study confirmed that miR-143-3p directly targets the MyD88 promoter and negatively regulates its expression, suggesting that the TLR pathway connected by MyD88 may be the possible upstream pathway of miR-143-3p regulating pyroptosis.

Intestinal I/R injury mainly involves inflammation and activation of thrombotic cascade reactions[36]. I/R injury of intestinal epithelium results in accumulation of damage-associated molecular patterns (DAMPs) in lamina propria[37]. When DAMPs bind to TLR on the cell surface, it activates transcription factors such as nuclear factor $\mathrm{KB}$ (NF-KB) to promote the upregulation of proteins associated with activation of innate immune responses[38]. A large number of studies have demonstrated that regulating the TLR4/MyD88/NF-KB pathway can alleviate I/R injury[39-41]. In this study, the protein expressions of TLR4, MyD88 and p-NF-KB/NF-KB in the OGD/R and I/R model group were significantly increased, while BMSC-exo significantly inversed the increase. And the further study showed that miR-143-3p can significantly regulate the expression of TLR4/MyD88/NF-KB pathway, suggesting that BMSC-exo may alleviate intestinal I/R injury by regulating TLR4/MyD88/NF-KB pathway through miR-143-3p. In addition, NF-KB induces the expression of pro-inflammatory cytokines to promote the inflammatory response[42]. NLRP3, a member of the inflammasome NLR family, is the core of inflammatory response and can activate caspase-1 to mediate pyroptosis[43]. Therefore, the TLR4/MyD88/NF-kB pathway may regulate NLRP3 to promote caspase-1-induced pyroptosis, and play an important role in the alleviation of intestinal I/R injury by BMSC-exo. The present study found that the changes of the miR-143-3p expression significantly altered the protein expressions of TLR4/MyD88/NF-KB pathway and pyroptosis pathway, suggesting that BMSC-exo regulated pyroptosis to alleviate intestinal I/R injury through miR143-3p mediated TLR4/MyD88/NF-KB pathway.

\section{Conclusion}

In conclusion, this study found that BMSC-exo has a good alleviating effect on intestinal I/R injury, which can increase cell viability and reduce apoptosis of OGD/R cell model in vitro, and improve the intestinal mucosal barrier of I/R mice model in vivo. And BMSC-exo can relieve inflammatory response and regulate the NLRP3-related pyroptosis pathway both in vitro and in vivo. In addition, sequencing of miRNA microarray showed that miR-143-3p plays an important role in BMSC-exo regulated intestinal I/R injury, and miR-143-3p directly targets MyD88 and negatively regulates its expression. Further studies confirmed that BMSC-exo regulated pyroptosis to alleviate intestinal I/R injury through miR-143-3p/MyD88/NFKB/NLRP3 axis.

\section{Declarations}




\section{Ethics approval and consent to participate}

All experimental protocols of the present study were approved by the First Hospital of Lanzhou University (No. LDYYLL2022-27) and all efforts were made to minimize animal suffering and reduce the number of animals used.

\section{Consent for publication}

Not Applicable

\section{Availability of data and materials}

The datasets used and/or analyzed during the current study are available from the corresponding author on reasonable request.

\section{Competing interests}

The authors declare that they have no conflict of interest.

\section{Funding}

This work was supported by the National Natural Science Foundation of China (No. 81960345), Natural Science Foundation of Gansu Province (No. 21JR11RA097) and the Foundation of First Hospital of Lanzhou University (NO. Idyyyn2020-18).

\section{Authors' contributions}

WZH and ZGR conceived and designed the research. WZH and ZGR performed most of the experiments. ZY, LJP and GWW performed parts of the experiments. WZH and YY analyzed the data. WZH, ZGR and LYF wrote the manuscript. All authors agree to be held accountable for all aspects of the research and confirm that the data are accurate. All authors reviewed the manuscript.

\section{Acknowledgements}

Not Applicable

\section{References}

1. Kalogeris, T., C. P. Baines, M. Krenz, and R. J. Korthuis. 2016. Ischemia/Reperfusion Comprehensive Physiology 7: 113-170. doi:10.1002/cphy.c160006.

2. Golts, E., and M. Onaitis. 2021. Commentary: Ischemia reperfusion-Looking ahead The Journal of thoracic and cardiovascular surgery 161:e124-e125 doi:10.1016/j.jtcvs.2019.12.010.

3. Mallick, I. H., W. Yang, M. C. Winslet, and A. M. Seifalian. 2004. Ischemia-reperfusion injury of the intestine and protective strategies against injury Digestive. diseases and sciences 49: 1359-1377. 
doi:10.1023/b:ddas.0000042232.98927.91.

4. Leenarts, C. A., J. Grootjans, I. H. Hundscheid, D. H. Schellekens, K. Lenaerts, W. A. Buurman, C. H. Dejong, and J. P. Derikx. 2019. Histopathology of human small intestinal and colonic ischemiareperfusion: Experiences from human IR-models. Histology and histopathology 34: 711-722. doi:10.14670/hh-18-074.

5. Barzegar, M., G. Kaur, F. N. E. Gavins, Y. Wang, C. J. Boyer, and J. S. Alexander. 2019. Potential therapeutic roles of stem cells in ischemia-reperfusion injury. Stem cell research 37: 101421. doi:10.1016/j.scr.2019.101421.

6. Miceli, V., M. Bulati, G. Iannolo, G. Zito, A. Gallo, and P. G. Conaldi. 2021. Therapeutic Properties of Mesenchymal Stromal/Stem Cells: The Need of Cell Priming for Cell-Free Therapies in Regenerative Medicine International journal of molecular sciences 22 doi:10.3390/ijms22020763.

7. Oliva, J. 2019. Therapeutic Properties of Mesenchymal Stem Cell on Organ Ischemia-Reperfusion Injury International journal of molecular sciences 20 doi:10.3390/ijms20215511.

8. Suh, J. H., H. S. Joo, E. B. Hong, H. J. Lee, and J. M. Lee. 2021. Therapeutic Application of Exosomes in Inflammatory Diseases International journal of molecular sciences 22 doi:10.3390/ijms22031144.

9. Liu, J., T. Chen, P. Lei, X. Tang, and P. Huang. 2019. Exosomes Released by Bone Marrow Mesenchymal Stem Cells Attenuate Lung Injury Induced by Intestinal Ischemia Reperfusion via the TLR4/NF-KB. Pathway International journal of medical sciences 16: 1238-1244. doi:10.7150/ijms.35369.

10. Kovacs, S. B., and E. A. Miao. 2017. Gasdermins: Effectors of Pyroptosis Trends. in cell biology 27: 673-684. doi:10.1016/j.tcb.2017.05.005.

11. Tsuchiya, K. 2021. Switching from Apoptosis to Pyroptosis: Gasdermin-Elicited Inflammation and Antitumor Immunity International journal of molecular sciences 22 doi:10.3390/ijms22010426.

12. Wree, A., A. Eguchi, M. D. McGeough, C. A. Pena, C. D. Johnson, A. Canbay, H. M. Hoffman, and A. E. Feldstein. 2014. NLRP3 inflammasome activation results in hepatocyte pyroptosis, liver inflammation, and fibrosis in mice Hepatology (Baltimore. Md) 59: 898-910. doi:10.1002/hep.26592.

13. Zhao, Y., J. Shi, and F. Shao. 2018. Inflammatory Caspases: Activation and Cleavage of Gasdermin-D In Vitro and During Pyroptosis Methods in molecular biology. (Clifton, NJ) 1714: 131-148. doi:10.1007/978-1-4939-7519-8_9.

14. Popov, S. V., L. N. Maslov, N. V. Naryzhnaya, A. V. Mukhomezyanov, A. V. Krylatov, S. Y. Tsibulnikov, V. V. Ryabov, M. V. Cohen, and J. M. Downey. 2021. The Role of Pyroptosis in Ischemic and Reperfusion Injury of the Heart. Journal of cardiovascular pharmacology and therapeutics 26: 562-574. doi:10.1177/10742484211027405.

15. Xu, X. N., Y. Jiang, L. Y. Yan, S. Y. Yin, Y. H. Wang, S. B. Wang, L. H. Fang, and G. H. Du. 2021. Aesculin suppresses the NLRP3 inflammasome-mediated pyroptosis via the Akt/GSK3ß/NF-KB pathway to mitigate myocardial ischemia/reperfusion injury. Phytomedicine: international journal of phytotherapy and phytopharmacology 92: 153687. doi:10.1016/j.phymed.2021.153687. 
16. Zeng, Q., Y. Zhou, D. Liang, H. He, X. Liu, R. Zhu, M. Zhang, X. Luo, Y. Wang, and G. Huang. 2020. Exosomes Secreted From Bone Marrow Mesenchymal Stem Cells Attenuate Oxygen-Glucose Deprivation/Reoxygenation-Induced Pyroptosis in PC12 Cells by Promoting AMPK-Dependent Autophagic Flux Frontiers in cellular neuroscience 14:182 doi:10.3389/fncel.2020.00182.

17. Liu, X., M. Zhang, H. Liu, R. Zhu, H. He, Y. Zhou, Y. Zhang, C. Li, D. Liang, Q. Zeng, and G. Huang. 2021. Bone marrow mesenchymal stem cell-derived exosomes attenuate cerebral ischemia-reperfusion injury-induced neuroinflammation and pyroptosis by modulating microglia M1/M2 phenotypes Experimental neurology 341:113700 doi:10.1016/j.expneurol.2021.113700.

18. Chiu, C. J., A. H. McArdle, R. Brown, H. J. Scott, and F. N. Gurd. 1970. Intestinal mucosal lesion in lowflow states. I. A morphological, hemodynamic, and metabolic reappraisal Archives of surgery (Chicago, Ill: 1960) 101:478 - 83 doi:10.1001/archsurg.1970.01340280030009.

19. Chen, F., D. Wang, X. Li, and H. Wang. 2020. Molecular Mechanisms Underlying Intestinal Ischemia/Reperfusion Injury: Bioinformatics Analysis and In Vivo Validation Medical science monitor: international. medical journal of experimental and clinical research 26: e927476. doi:10.12659/msm.927476.

20. Spees, J. L., R. H. Lee, and C. A. Gregory. 2016. Mechanisms of mesenchymal stem/stromal cell function Stem cell research \& therapy 7:125 doi:10.1186/s13287-016-0363-7.

21. Yu, B., X. Zhang, and X. Li. 2014. Exosomes derived from mesenchymal stem cells. International journal of molecular sciences 15: 4142-4157. doi:10.3390/ijms15034142.

22. Phinney, D. G., and M. F. Pittenger. 2017. Concise Review: MSC-Derived Exosomes for Cell-Free Therapy Stem cells (Dayton. Ohio) 35: 851-858. doi:10.1002/stem.2575.

23. Li, X., Y. Zhang, Y. Wang, D. Zhao, C. Sun, S. Zhou, D. Xu, and J. Zhao. 2020. Exosomes Derived from CXCR4-Overexpressing BMSC Promoted Activation of Microvascular Endothelial Cells in Cerebral Ischemia/Reperfusion Injury Neural plasticity 2020:8814239 doi:10.1155/2020/8814239.

24. Chen, Q., Y. Liu, X. Ding, Q. Li, F. Qiu, M. Wang, Z. Shen, H. Zheng, and G. Fu. 2020. Bone marrow mesenchymal stem cell-secreted exosomes carrying microRNA-125b protect against myocardial ischemia reperfusion injury via targeting SIRT7 Molecular and cellular biochemistry 465:103-114 doi:10.1007/s11010-019-03671-z.

25. Shi, J., W. Gao, and F. Shao. 2017. Pyroptosis: Gasdermin-Mediated Programmed Necrotic Cell Death Trends in biochemical sciences 42:245-254 doi:10.1016/j.tibs.2016.10.004.

26. Broz, P. 2015. Immunology: Caspase target drives pyroptosis. Nature 526: 642-643. doi:10.1038/nature15632.

27. Jia, C., H. Chen, J. Zhang, K. Zhou, Y. Zhuge, C. Niu, J. Qiu, X. Rong, Z. Shi, J. Xiao, Y. Shi, and M. Chu. 2019. Role of pyroptosis in cardiovascular diseases International immunopharmacology 67: 311318. doi:10.1016/j.intimp.2018.12.028.

28. Zheng, Z., and G. Li. 2020. Mechanisms and Therapeutic Regulation of Pyroptosis in Inflammatory Diseases and Cancer International journal of molecular sciences 21 doi:10.3390/ijms21041456. 
29. Qiu, Z., S. Lei, B. Zhao, Y. Wu, W. Su, M. Liu, Q. Meng, B. Zhou, Y. Leng, and Z. Y. Xia. 2017. NLRP3 Inflammasome Activation-Mediated Pyroptosis Aggravates Myocardial Ischemia/Reperfusion Injury in Diabetic Rats Oxidative medicine and cellular longevity 2017:9743280 doi:10.1155/2017/9743280.

30. Wu, M. Y., G. T. Yiang, W. T. Liao, A. P. Tsai, Y. L. Cheng, P. W. Cheng, C. Y. Li, and C. J. Li. 2018. Current Mechanistic Concepts in Ischemia and Reperfusion Injury Cellular physiology and biochemistry: international journal of experimental cellular physiology, biochemistry. and pharmacology 46: 16501667. doi:10.1159/000489241.

31. Pegtel, D. M., and S. J. Gould. 2019. Exosomes Annual review of biochemistry 88: 487-514. doi:10.1146/annurev-biochem-013118-111902.

32. Dexheimer, P. J., and L. Cochella. 2020. MicroRNAs: From Mechanism to Organism. Frontiers in cell and developmental biology 8: 409. doi:10.3389/fcell.2020.00409.

33. Ghafouri-Fard, S., H. Shoorei, M. Taheri.. Biomedicine. 2020. Non-coding RNAs participate in the ischemia-reperfusion injury. \& pharmacotherapy = Biomedecine \& pharmacotherapie 129: 110419. doi:10.1016/j.biopha.2020.110419.

34. Chen, G., M. Wang, Z. Ruan, L. Zhu, and C. Tang. 2021. Mesenchymal stem cell-derived exosomal miR-143-3p suppresses myocardial ischemia-reperfusion injury by regulating autophagy. Life sciences 280: 119742. doi:10.1016/j.Ifs.2021.119742.

35. Deguine, J., and G. M. Barton. 2014. MyD88: a central player in innate immune signaling F1000prime reports 6:97 doi:10.12703/p6-97.

36. Subramanian, S., H. Geng, and X. D. Tan. 2020. Cell death of intestinal epithelial cells in intestinal diseases Sheng li xue bao. [Acta physiologica Sinica]72: 308-324.

37. Donovan, C., G. Liu, S. Shen, J. E. Marshall, R. Y. Kim, C. A. Alemao, K. F. Budden, J. P. Choi, M. Kohonen-Corish, E. M. El-Omar, I. A. Yang, and P. M. Hansbro. 2020. The role of the microbiome and the NLRP3 inflammasome in the gut and lung. Journal of leukocyte biology 108: 925-935. doi:10.1002/jlb.3mr0720-472rr.

38. Nadatani, Y., T. Watanabe, S. Shimada, K. Otani, T. Tanigawa, and Y. Fujiwara. 2018. Microbiome and intestinal ischemia/reperfusion injury. Journal of clinical biochemistry and nutrition 63: 26-32. doi:10.3164/jcbn.17-137.

39. Li, S., L. Luo, Y. He, R. Li, Y. Xiang, Z. Xing, Y. Li, A. A. Albashari, X. Liao, K. Zhang, L. Gao, and Q. Ye. 2021. Dental pulp stem cell-derived exosomes alleviate cerebral ischaemia-reperfusion injury through suppressing inflammatory response. Cell proliferation 54: e13093. doi:10.1111/cpr.13093.

40. Du, Y., B. Qian, L. Gao, P. Tan, H. Chen, A. Wang, T. Zheng, S. Pu, X. Xia, and W. Fu. 2019. Aloin Preconditioning Attenuates Hepatic Ischemia/Reperfusion Injury via Inhibiting TLR4/MyD88/NF-KB Signal Pathway In Vivo and In Vitro Oxidative medicine and cellular longevity 2019:3765898 doi:10.1155/2019/3765898.

41. Zhang, W., J. Song, W. Li, D. Kong, Y. Liang, X. Zhao, and G. Du. 2020. Salvianolic Acid D Alleviates Cerebral Ischemia-Reperfusion Injury by Suppressing the Cytoplasmic Translocation and Release of 
HMGB1-Triggered NF-kB Activation to Inhibit Inflammatory Response Mediators of inflammation 2020:9049614 doi:10.1155/2020/9049614.

42. Chawla, M., P. Roy, and S. Basak. 2021. Role of the NF-KB system in context-specific tuning of the inflammatory gene response. Current opinion in immunology 68: 21-27. doi:10.1016/j.coi.2020.08.005.

43. He, Y., H. Hara, and G. Núñez. 2016. Mechanism and Regulation of NLRP3 Inflammasome Activation Trends in biochemical sciences 41:1012-1021 doi:10.1016/j.tibs.2016.09.002.

\section{Figures}

A

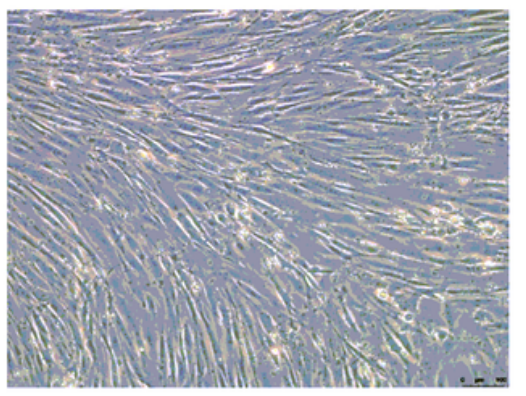

B

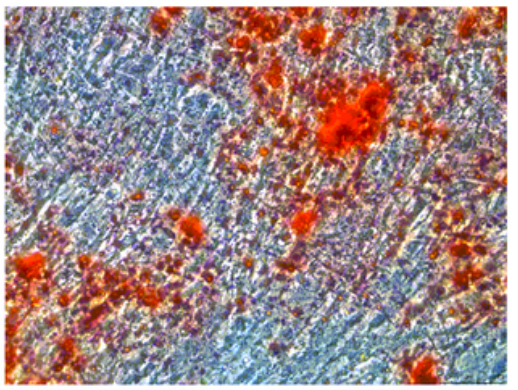

C

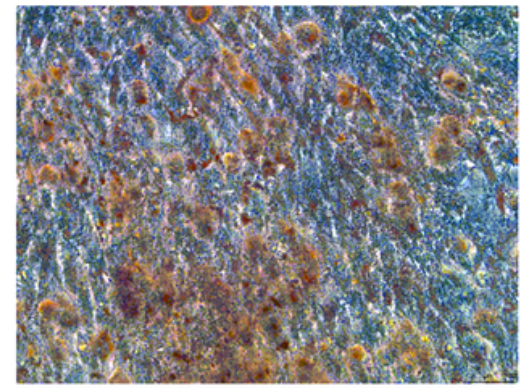

D
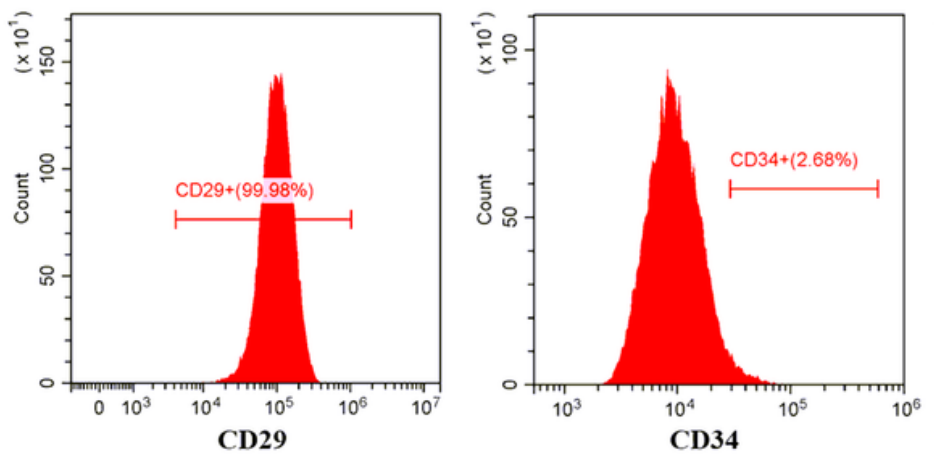

F
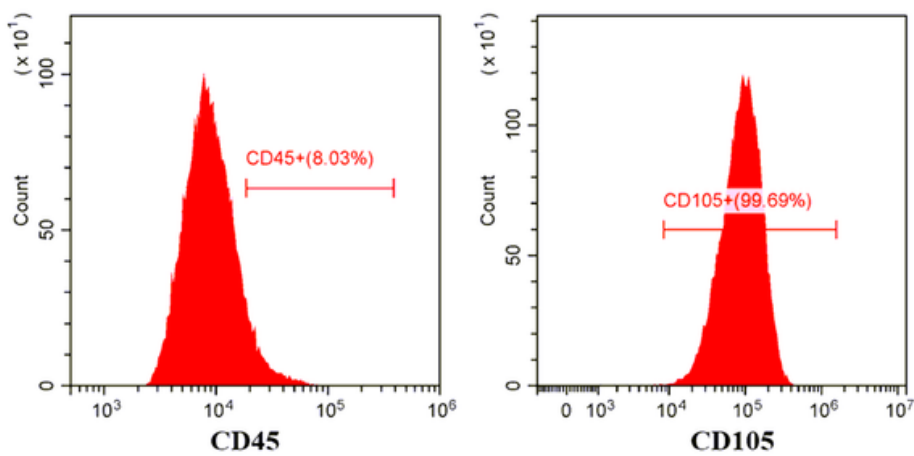

$\mathbf{E}$

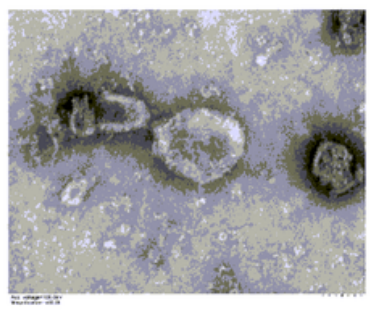

G

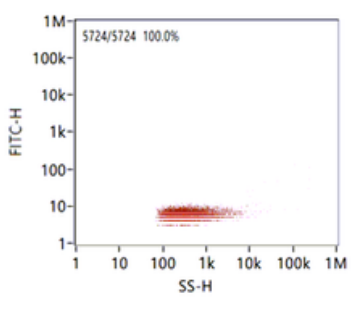

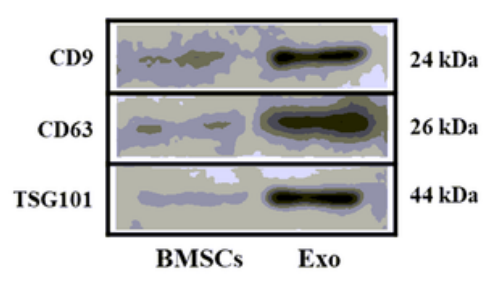

Figure 1

Identification of BMSCs and BMSC-exo. (A) The morphology of third-generation BMSCs was observed under a light microscope. Magnification, $\times 100$. (B) The osteogenic differentiation ability of BMSCs was analyzed by alizarin red staining. Magnification, $\times 100$. (C) Adipogenic differentiation ability of BMSCs was detected by oil red 0 staining. Magnification, $\times 100$. (D) Surface markers of CD29, CD34, CD45 and CD105 in BMSCs were detected by flow cytometry. (E) The morphology of BMSC-exo was observed by 
transmission electron microscopy (TEM). (F) The concentration and particle size of BMSC-exo were detected by nanoparticle tracking analysis (NTA). (G) Western blot analysis was used to determine the CD9, CD63 and TSG101 markers in BMSC-exo.

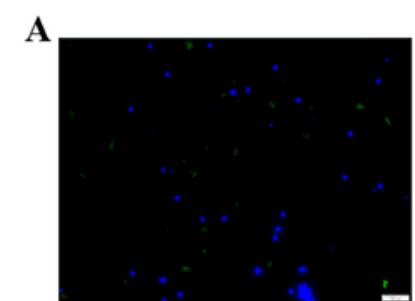

$6 \mathrm{~h}$

B

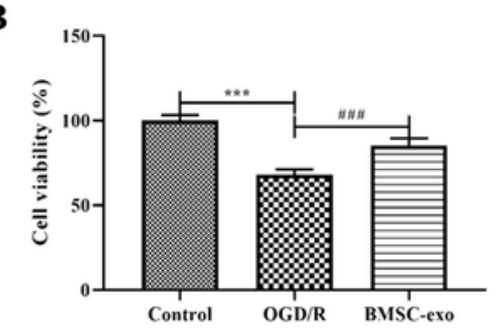

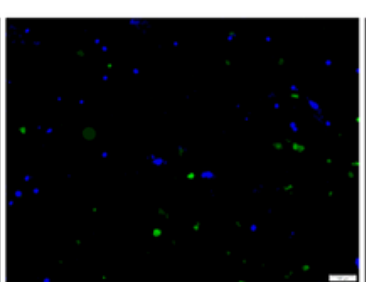

$12 \mathrm{~h}$

C

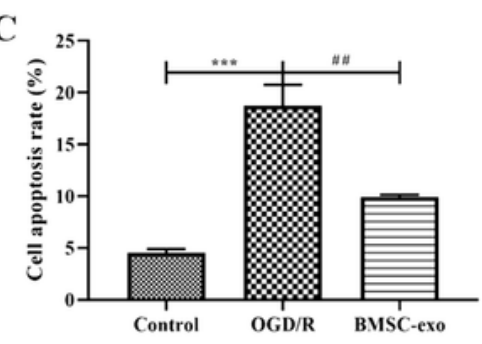

D
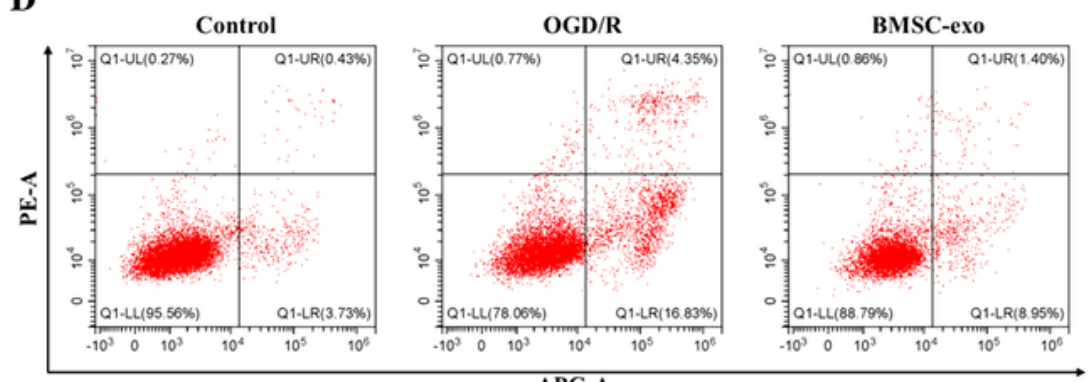

E
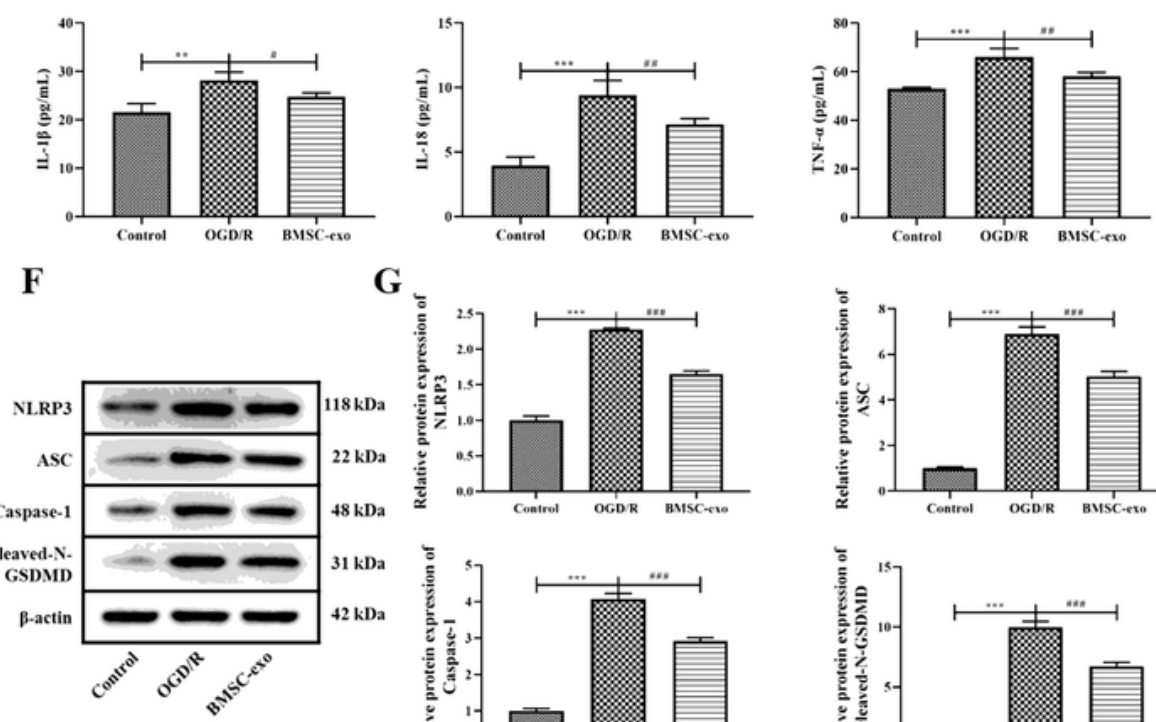

G
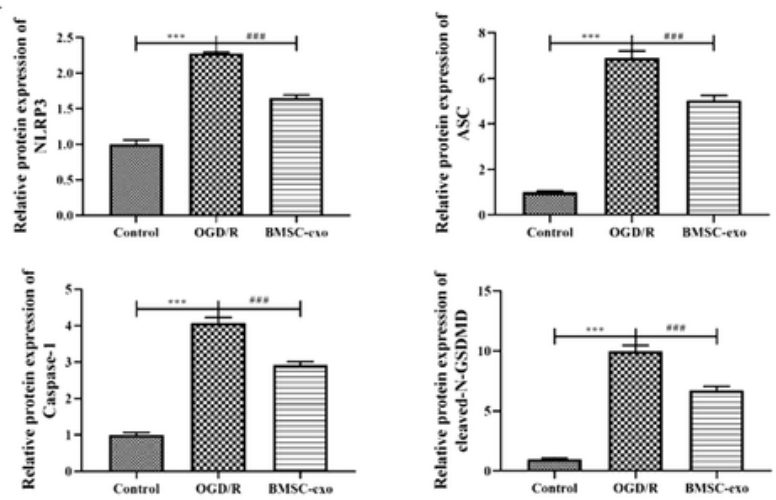

Figure 2 
Effects of BMSC-exo on OGD/R cell model. (A) Representative images of PKH-67 fluorescence-labeled BMSC-exo co-cultured with IEC- 6 cells for 6 h,12 h, and $24 \mathrm{~h}$. Magnification, $\times 100$. (B) Cells viability was detected by CCK-8 assay. (C, D) Cell apoptosis was detected by flow cytometry assay. (E) The expressions of inflammatory cytokines IL-1 $\beta$, IL-18 and TNF-a were detected by ELISA. (F) Protein expressions of NLRP3, ASC, Caspase-1 and cleaved-N-GSDMD were detected by western blot analysis. (G) Densitometry analysis of the protein expression. Data were shown as mean $\pm \mathrm{SD}$. ${ }^{*} \mathrm{P}<0.05,{ }^{* *} \mathrm{P}<0.01$ and ${ }^{* *} \mathrm{P}<$ 0.001 , compared with the control group; ${ }^{\#} \mathrm{P}<0.05$, ${ }^{\#} \mathrm{P}<0.01$ and ${ }^{\# \#} \mathrm{P}<0.001$, compared with the OGD/R model group. 
A
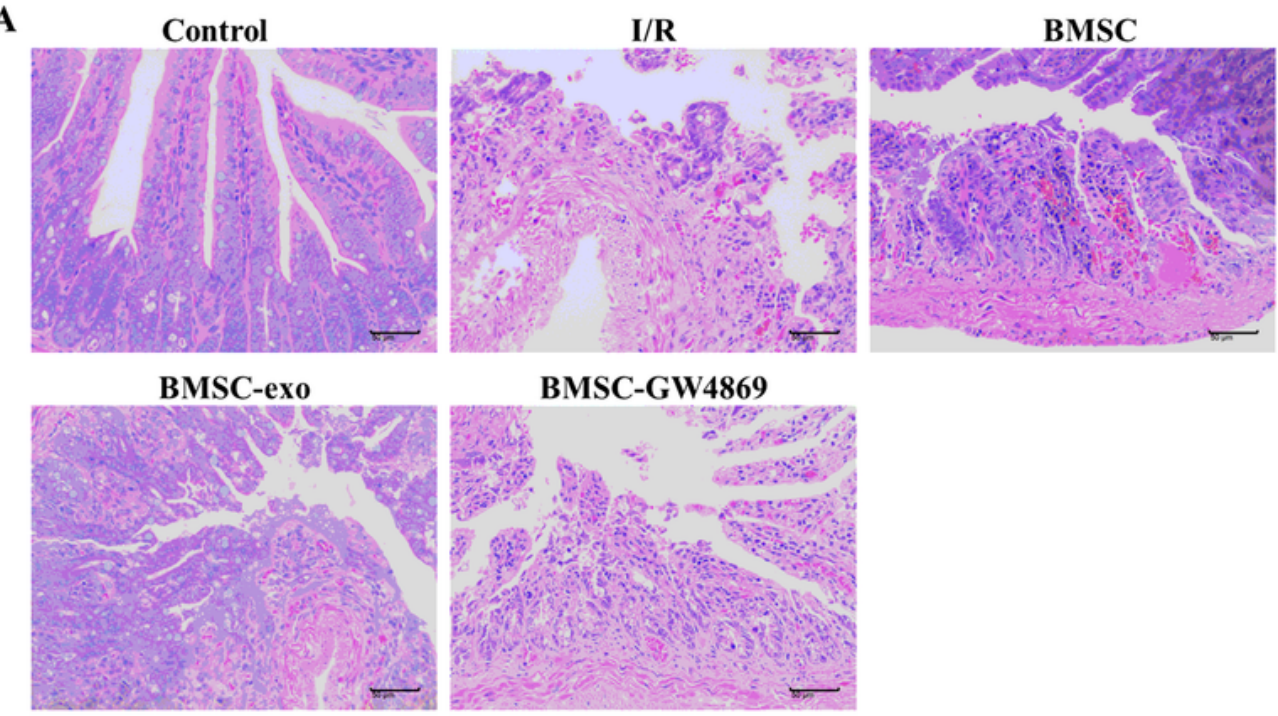

BMSC-GW4869

B

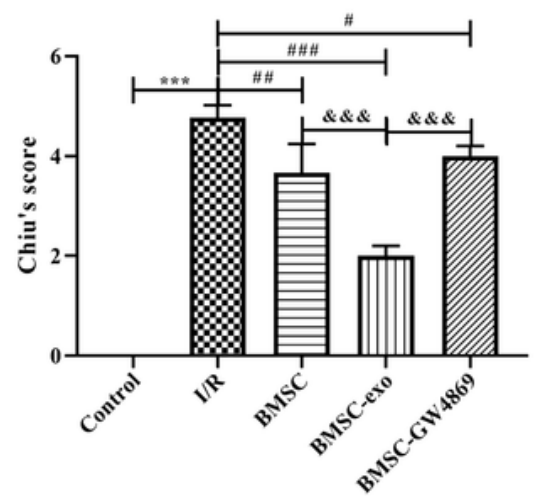

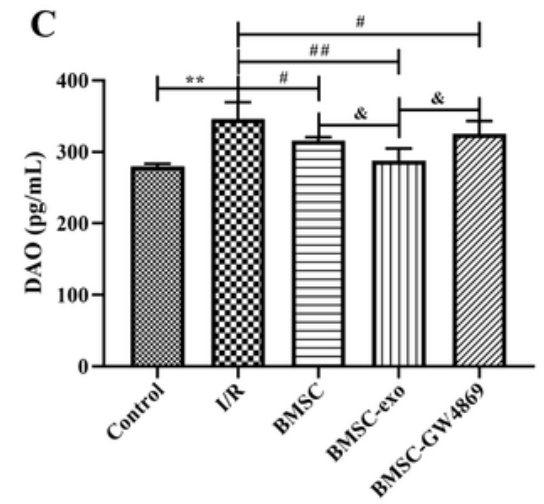

D

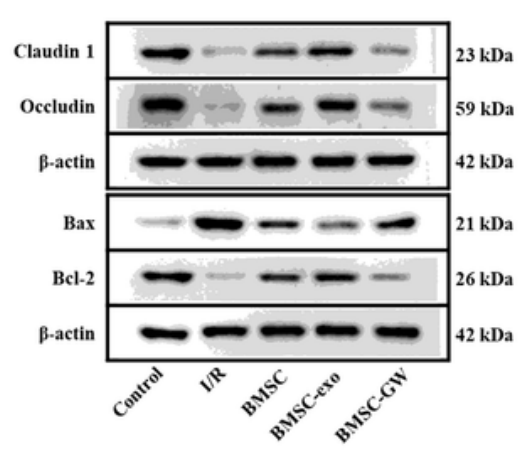

$\mathbf{E}$

\section{,}
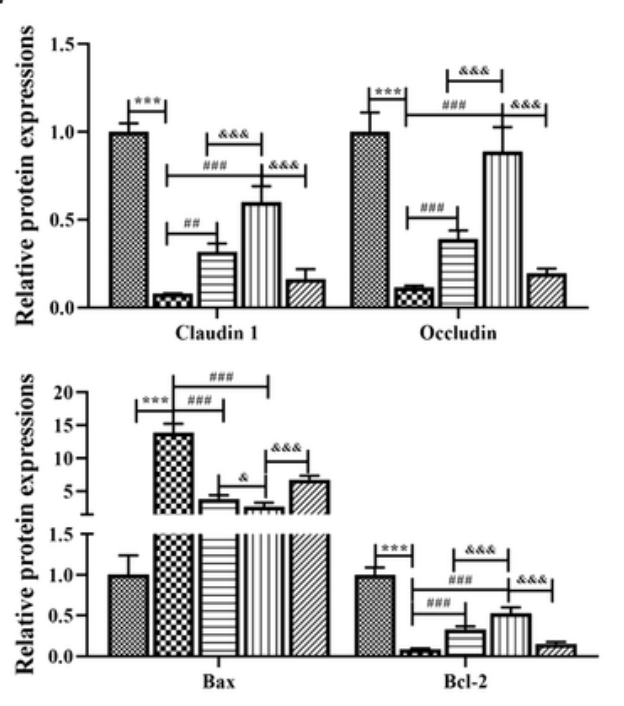

Control

I/R

口 BMSC

m BMSC-exo

m BMSC-GW4869

Control

Lod $\mathbf{R}$

口 BMSC

四 BMSC-exo

m BMSC-GW4869

\section{Figure 3}

Effects of BMSC-exo on intestinal barrier mucosal injury in I/R model mice. (A) Hematoxylin and eosin staining of mouse intestinal tissue. Magnifications, $\times 400$. (B) Chiu's score of HE staining was used to evaluate pathological lesions. (C) The expression level of DAO in serum was examined by ELISA. (D) Protein expressions of Claudin 1, Occludin, Bax and Bcl-2 were detected by western blot analysis. (E) Densitometry analysis of the protein expression. Data were shown as mean $\pm \mathrm{SD}$. ${ }^{*} \mathrm{P}<0.05,{ }^{* *} \mathrm{P}<0.01$ 
and ${ }^{* * *} \mathrm{P}<0.001$, compared with the control group; ${ }^{\#} \mathrm{P}<0.05$, ${ }^{\# \#} \mathrm{P}<0.01$ and ${ }^{\# \# \#} \mathrm{P}<0.001$, compared with the I/R model group; ${ }^{2} P<0.05,{ }^{\& \&} P<0.01$ and ${ }^{\& \& \&} P<0.001$, compared with the BMSC-exo group.
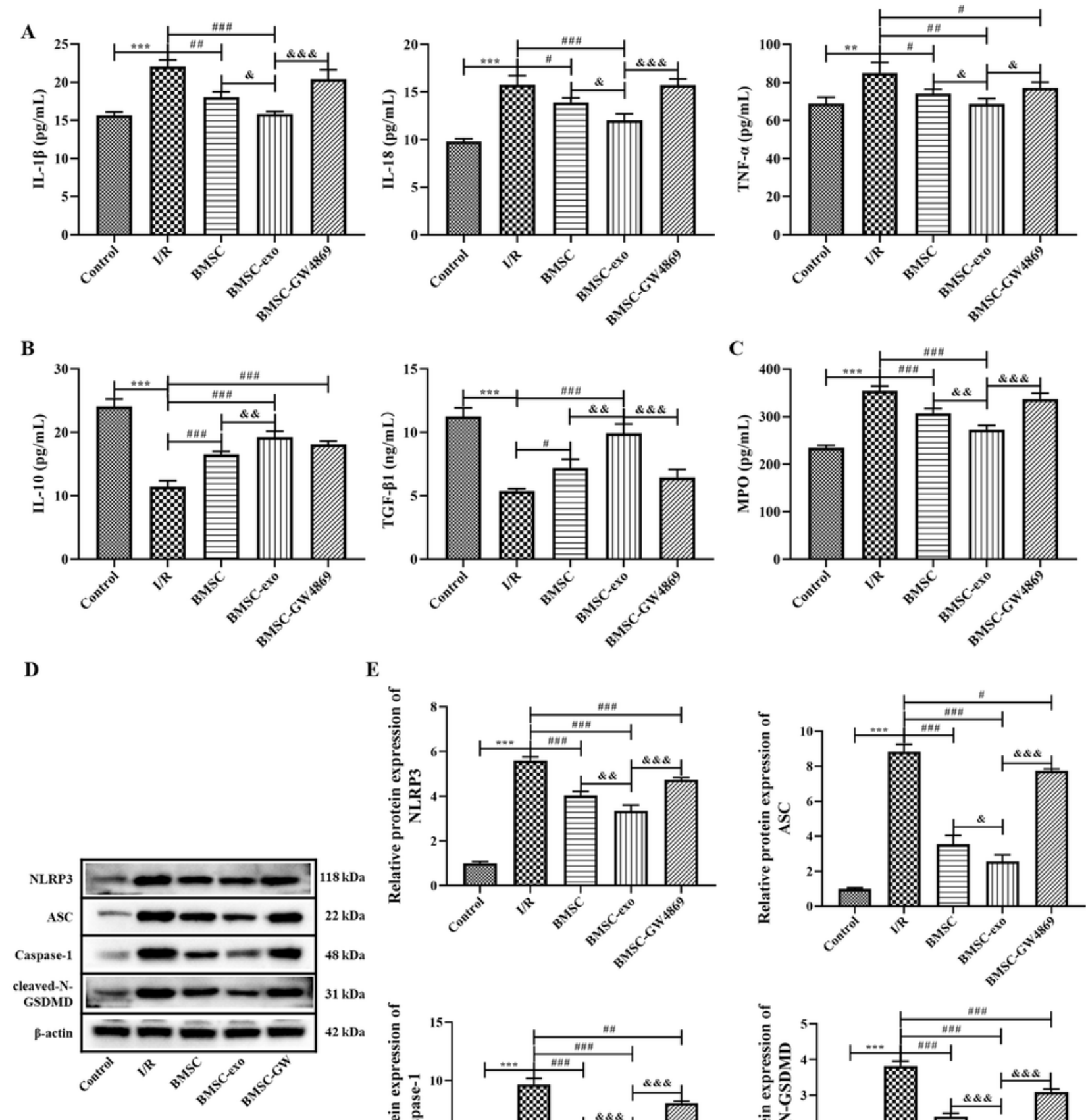

$\mathbf{E}$
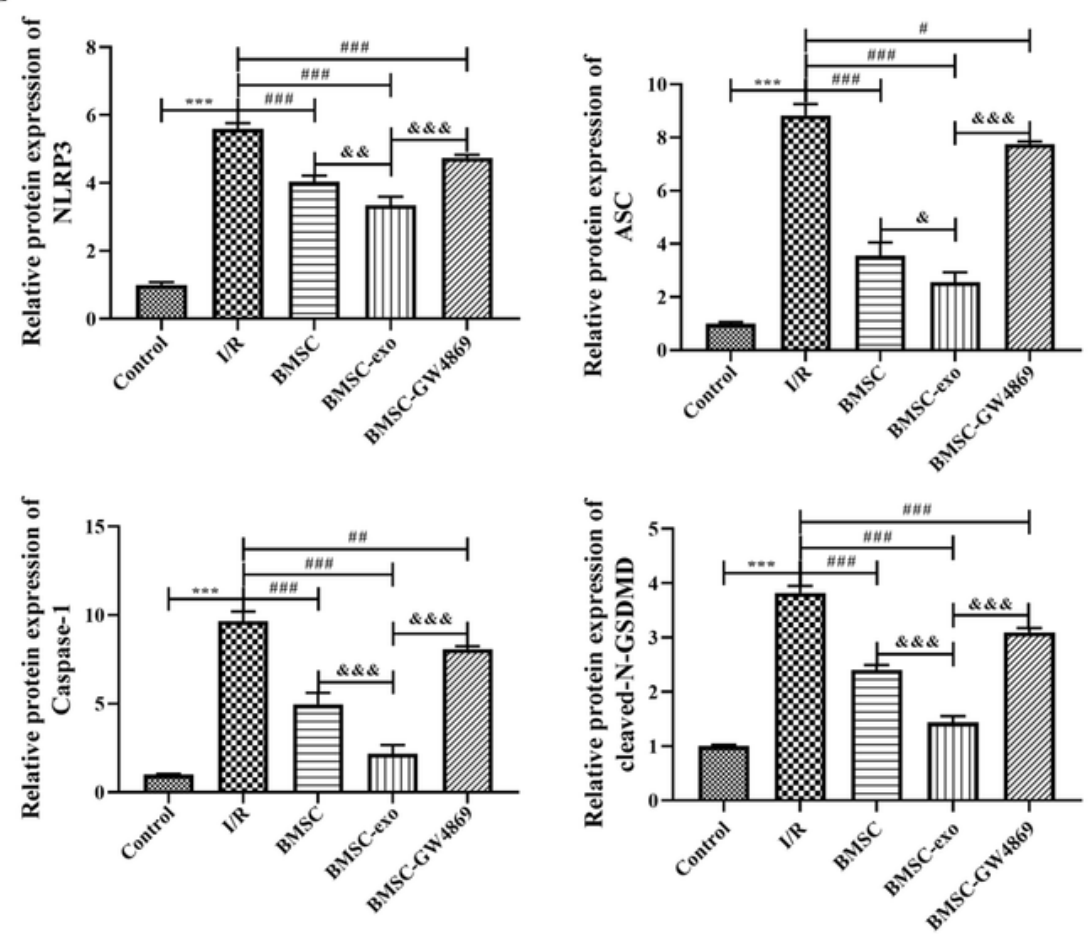

Figure 4

Effects of BMSC-exo on cytokines and pyroptosis-related proteins in I/R model mice. (A-C) The expression levels of IL-1 $\beta$, IL-18, TNF- $\alpha, I L-10, T$, TF- $\beta 1$, and MPO in serum were examined by ELISA. (D) 
Protein expressions of NLRP3, ASC, Casepase-1 and cleaved-N-GSDMD were detected by western blot analysis. (E) Densitometry analysis of the protein expression. Data were shown as mean $\pm S D$. ${ }^{*} P<0.05$, ${ }^{* *} \mathrm{P}<0.01$ and ${ }^{* *} \mathrm{P}<0.001$, compared with the control group; ${ }^{\#} \mathrm{P}<0.05$, ${ }^{\# \#} \mathrm{P}<0.01$ and ${ }^{\# \# \#} \mathrm{P}<0.001$, compared with the I/R model group; ${ }^{\&} P<0.05$, \& $P<0.01$ and ${ }^{\& \& \&} P<0.001$, compared with the BMSCexo group.

A

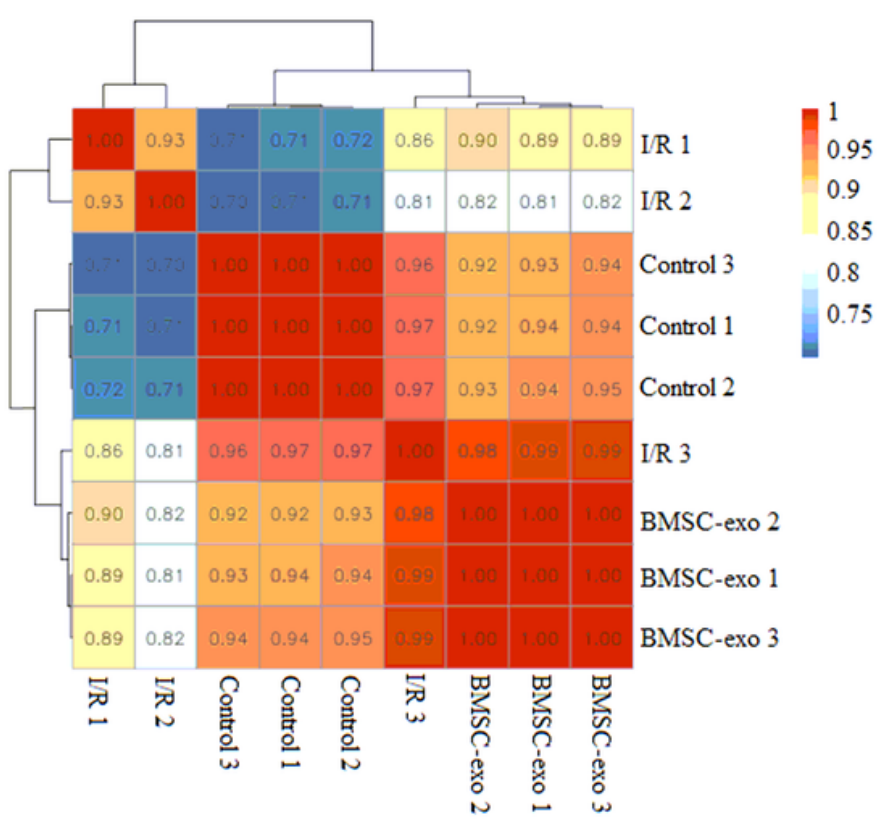

C

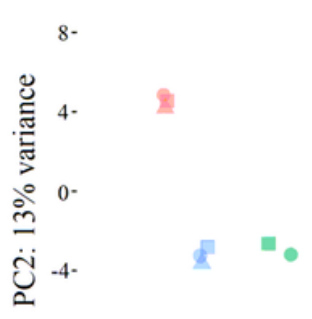

$\mathbf{E}$

Control vs BMSC-exo

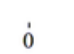

PC1: $67 \%$ variance

2́o

F
Replication

- rep 1

$\Delta$ rep 2

- rep 3

condition

- Control

- I/R

- BMSC-exo
B

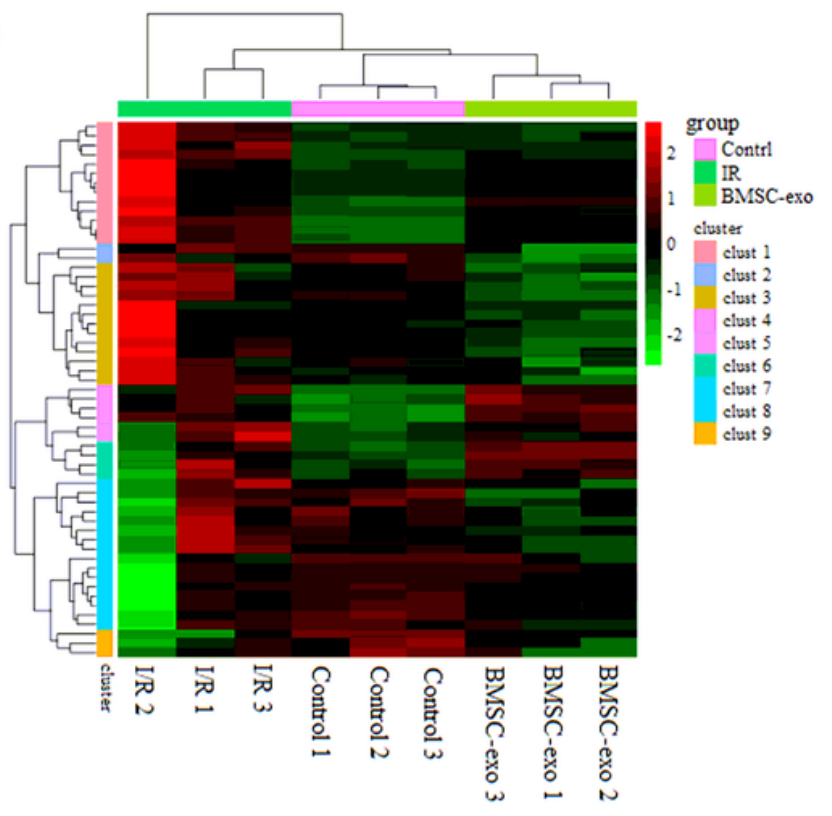

D

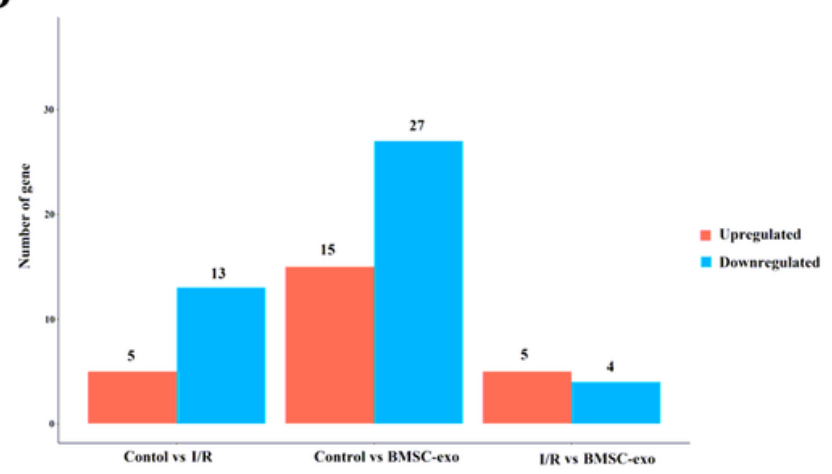

G
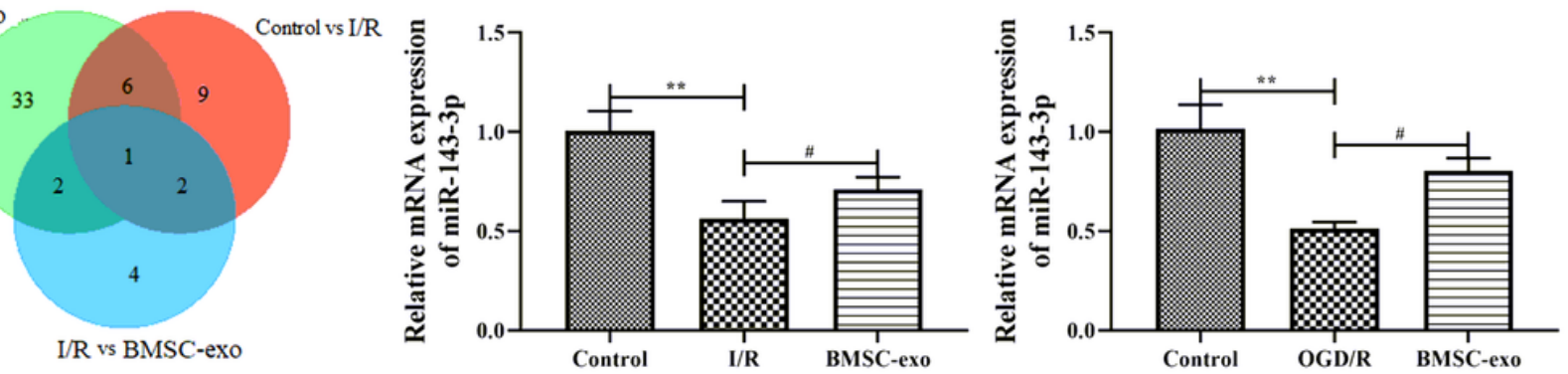

\section{Figure 5}

The miRNA expression profile of intestinal tissues in mice was detected by miRNA microarray. (A) Pearson correlation coefficient showed the correlation between miRNA expression levels among samples. The left and upper sides were sample clustering, the right and lower sides of the figure were sample 
names, and different colors represent the correlation between the two samples. (B) Cluster analysis of differentially expressed miRNA. Horizontal represents miRNA and each column represents one sample. Red represents miRNA with high expression and green represents miRNA with low expression. (C) Principal components analysis. The abscissa was the first principal component and the ordinate was the second principal component. Different shapes in the figure indicate different samples, and different colors indicate different groups. (D) Statistical analysis results of miRNA expression differences. The abscissa represents the comparison group for differential analysis, the ordinate represents the number of differential miRNAs, the red represents up-regulated, and the green represents down-regulated. (E) Venn diagram of miRNA differential expression. The overlapped miRNA was miR-143-3p. (F-G) The mRNA expression of miR-143-3p was detected by RT-PCR, and the relative expression was normalized to U6 expression. Data were shown as mean $\pm \mathrm{SD}$. ${ }^{*} \mathrm{P}<0.05$, ${ }^{* \star} \mathrm{P}<0.01$ and ${ }^{* \star *} \mathrm{P}<0.001$, compared with the control group; ${ }^{\#} \mathrm{P}<0.05$, \#\# $\mathrm{P}<0.01$ and ${ }^{\# \# \# ~} \mathrm{P}<0.001$, compared with the I/R or $\mathrm{OGD} / \mathrm{R}$ model group.

A

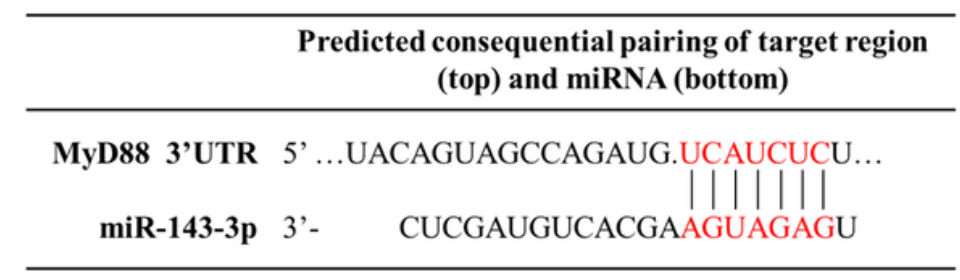

B

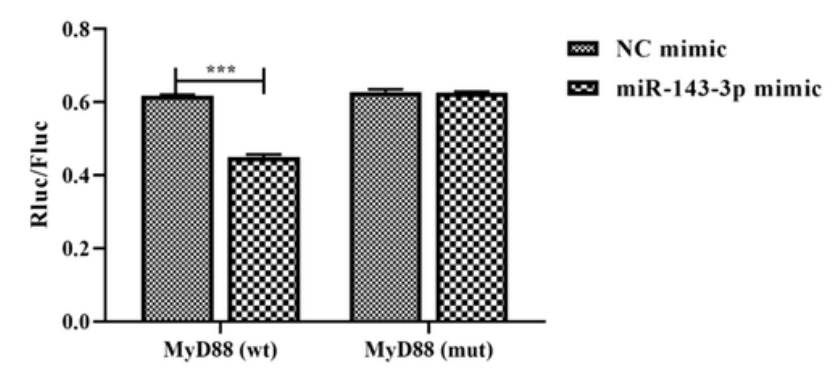

C

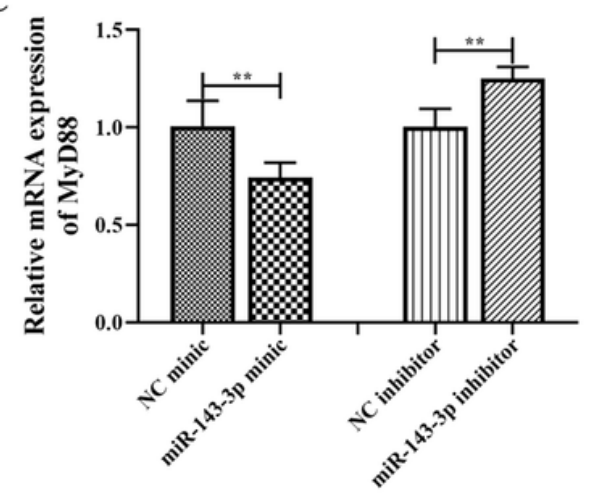

D

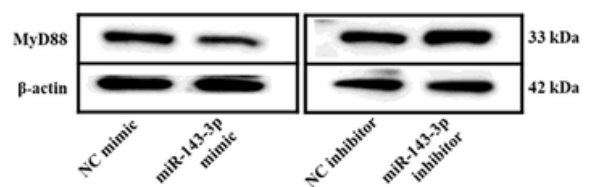

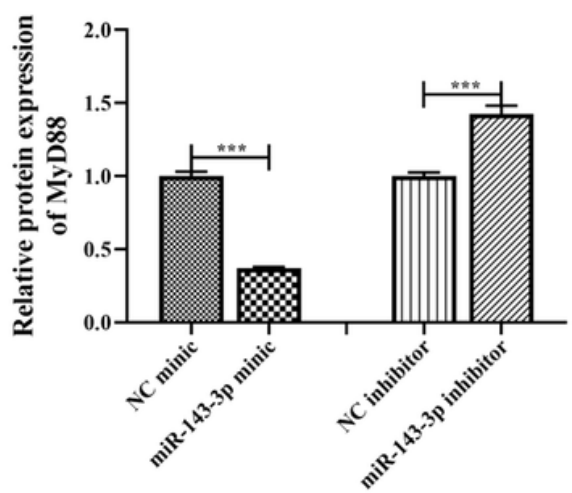

\section{Figure 6}

miR-143-3p directly targeted MyD88 and negatively modulated the expression of MyD88. (A) The target sites between miR-143-3p and 3'-UTR of MyD88 were predicted by bioinformatics websites. (B) The relative luciferase activity was detected by dual-luciferase reporter assay after transfection with miR-1433p mimic or NC mimic. ${ }^{* \star} \mathrm{P}<0.001$. (C) The mRNA expression of MyD88 was detected by RT-PCR, and the relative expression was normalized to Actin expression. (D) Protein expression of MyD88 was detected by western blot analysis. Data were shown as mean \pm SD. ${ }^{*} \mathrm{P}<0.05,{ }^{* *} \mathrm{P}<0.01$ and ${ }^{* \star *} \mathrm{P}<0.001$, compared with the NC-mimic or NC-inhibitor group. 
A

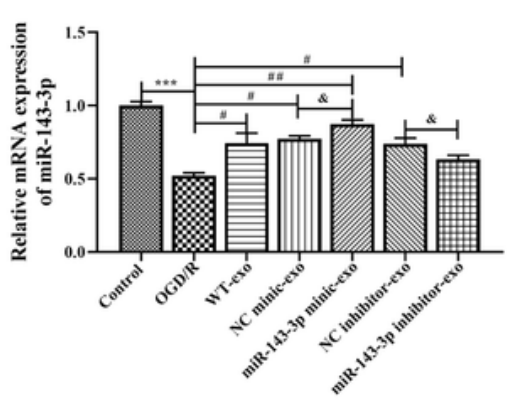

B

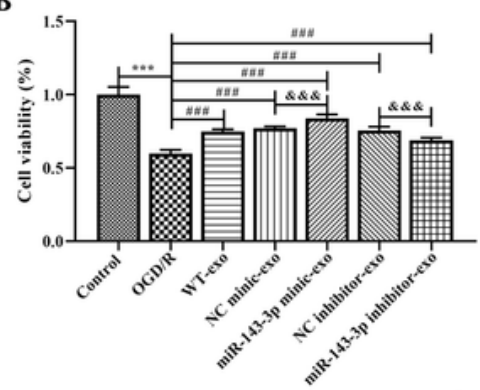

C

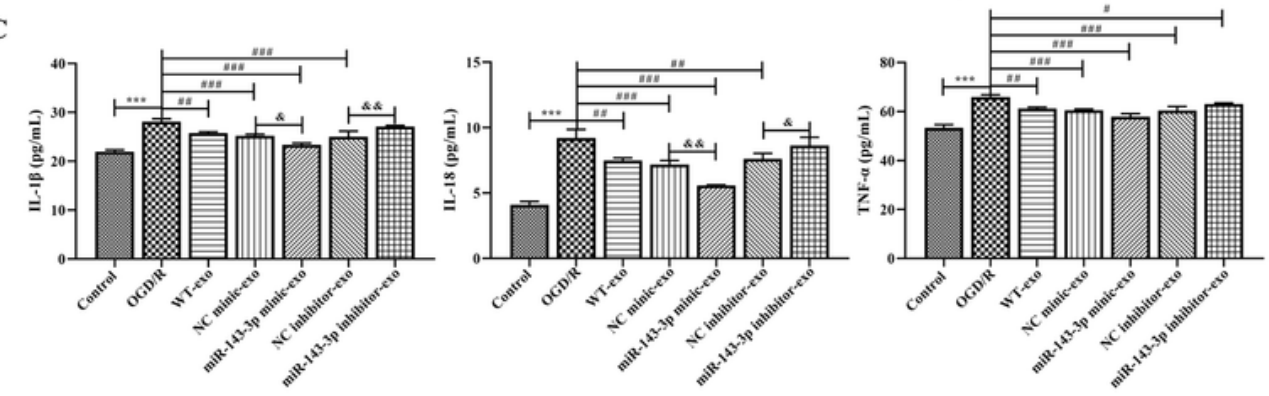

D
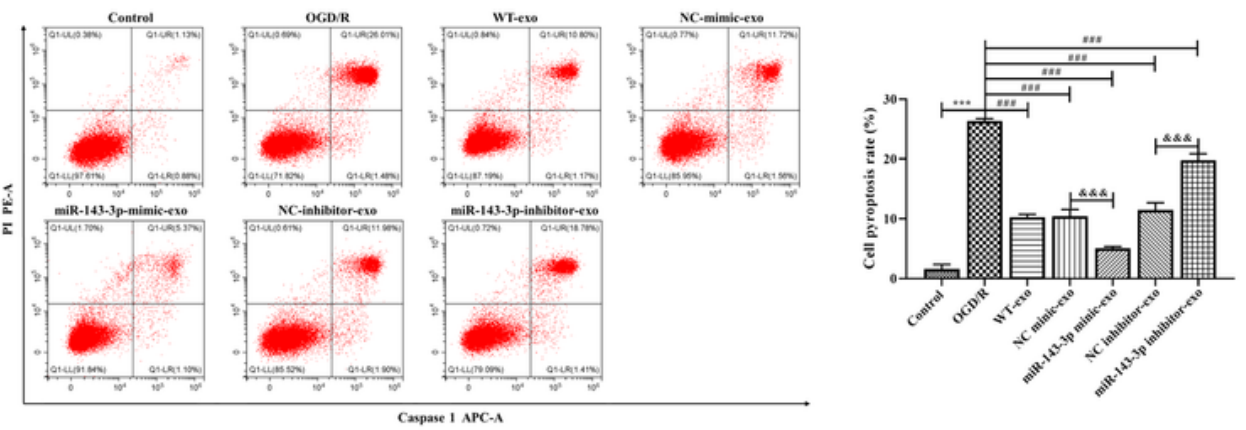

$\mathbf{E}$
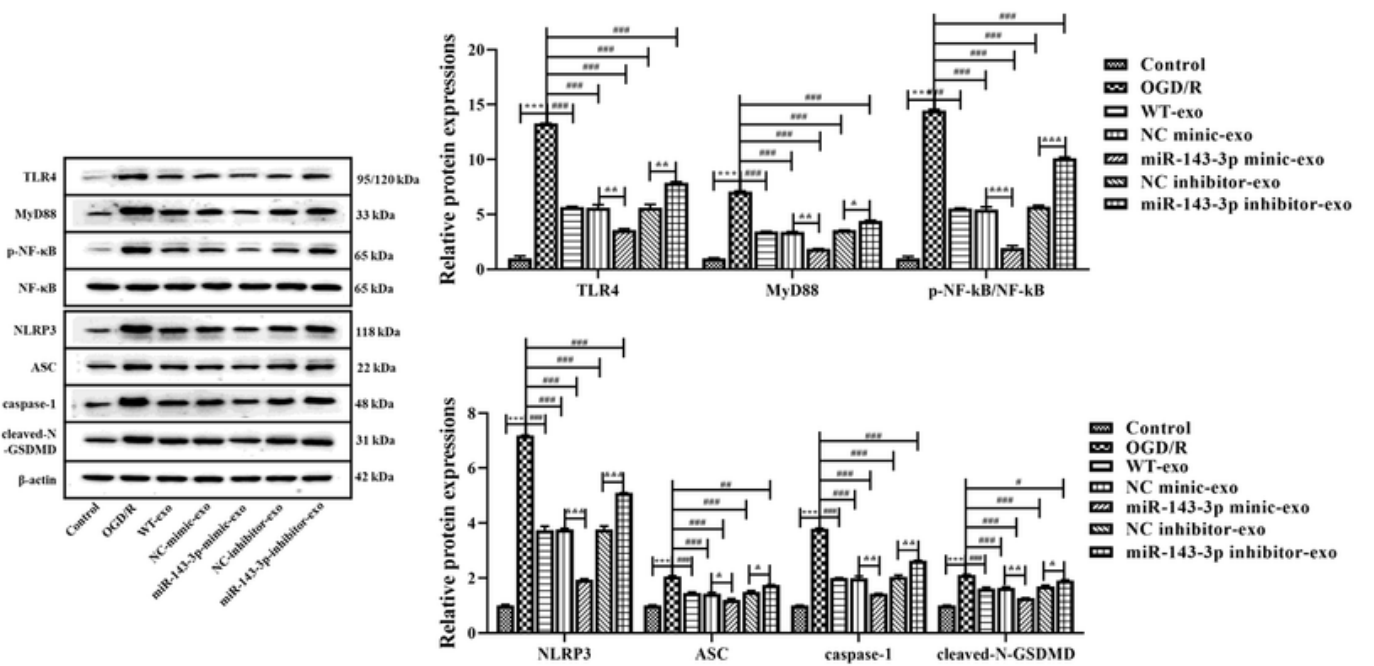

Figure 7

Effects of miR-143-3p in BMSC-exo on OGD/R cell model. (A) The mRNA expression of miR-143-3p was detected by RT-PCR, and the relative expression was normalized to U6 expression. (B) Cells viability was detected by CCK-8 assay. (C) The expressions of IL-1 $\beta$, IL-18 and TNF- $\alpha$ in cells were detected by ELISA. (D) Cell pyroptosis rate was detected by flow cytometry assay. (E) Protein expressions of TLR4, MyD88, pNF-кB, NF-kB, NLRP3, ASC, caspase-1 and cleaved-N-GSDMD were detected by western blot analysis. 
Data were shown as mean $\pm \mathrm{SD}$. ${ }^{*} \mathrm{P}<0.05,{ }^{* \star} \mathrm{P}<0.01$ and ${ }^{* \star} \mathrm{P}<0.001$, compared with the control group; ${ }^{\#} \mathrm{P}<0.05$, \#\# $\mathrm{P}<0.01$ and ${ }^{\# \# \#} \mathrm{P}<0.001$, compared with the 0 GD/R model group. ${ }^{\&} \mathrm{P}<0.05$, \&\& $\mathrm{P}<0.01$ and ${ }^{\text {\&\& }} \mathrm{P}<0.001$, compared with the NC mimic-exo or NC inhibitor-exo group.

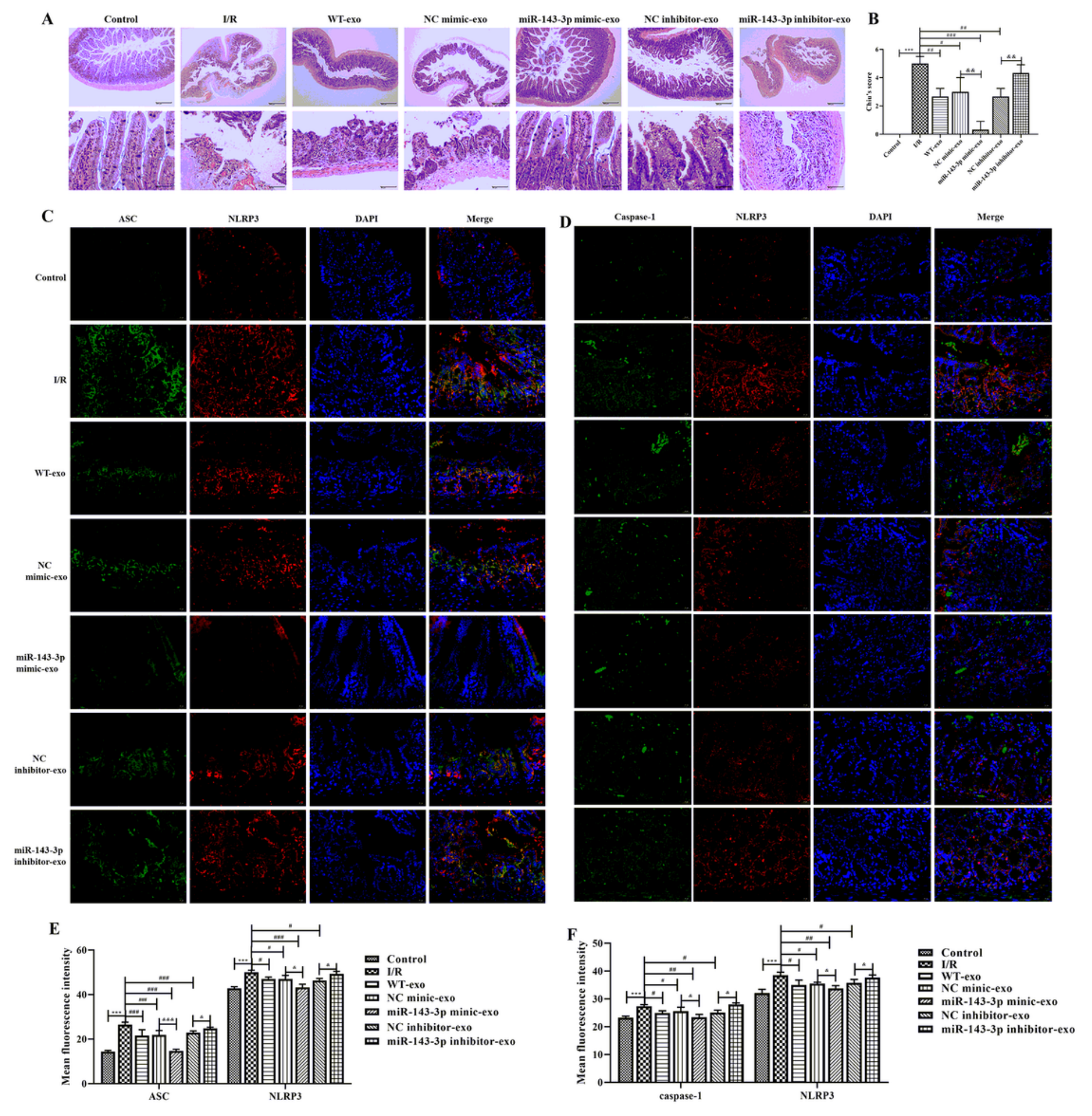

Figure 8 
Effects of miR-143-3p in BMSC-exo on I/R model mice. (A) Hematoxylin and eosin staining of mouse intestinal tissue. Magnifications, $\times 400$. (B) Chiu's score of HE staining was used to evaluate pathological lesions $\times 400$. (C) Representative images showing immunofluorescence staining of ASC (green) and NLRP3 (red) in intestinal tissues of mice. Nuclei were stained with 4',6-diamidino-2-phenylindole (blue). Magnification, $\times 400$. (D) Representative images showing immunofluorescence staining of caspase-1 (green) and NLRP3 (red) in intestinal tissues of mice. Nuclei were stained with 4',6-diamidino-2phenylindole (blue). Magnification, $\times 400$. (E) Mean fluorescence intensity in C. (F) Mean fluorescence intensity in D. Data were shown as mean $\pm \mathrm{SD} .{ }^{*} \mathrm{P}<0.05$, ${ }^{* *} \mathrm{P}<0.01$ and ${ }^{* *} \mathrm{P}<0.001$, compared with the control group; ${ }^{\#} \mathrm{P}<0.05$, ${ }^{\# \#} \mathrm{P}<0.01$ and ${ }^{\# \# \#} \mathrm{P}<0.001$, compared with the $\mathrm{I} / \mathrm{R}$ model group; \& $\mathrm{P}<0.05$, \&\& $P<0.01$ and ${ }^{\text {\&\& }} \mathrm{P}<0.001$, compared with the NC mimic-exo or NC inhibitor-exo group.
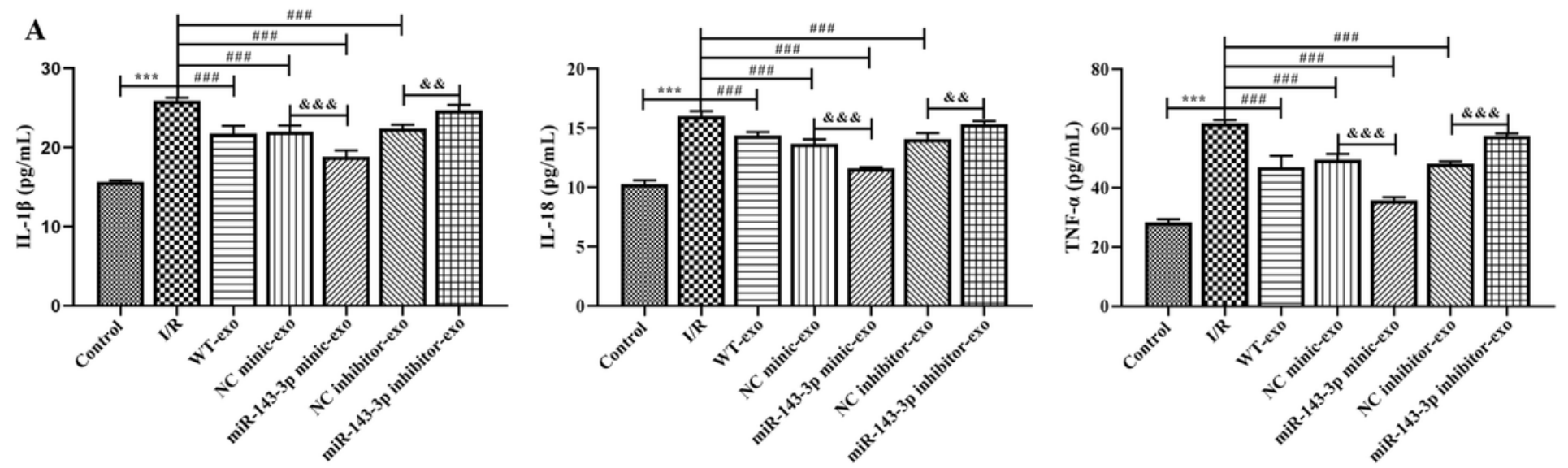

B

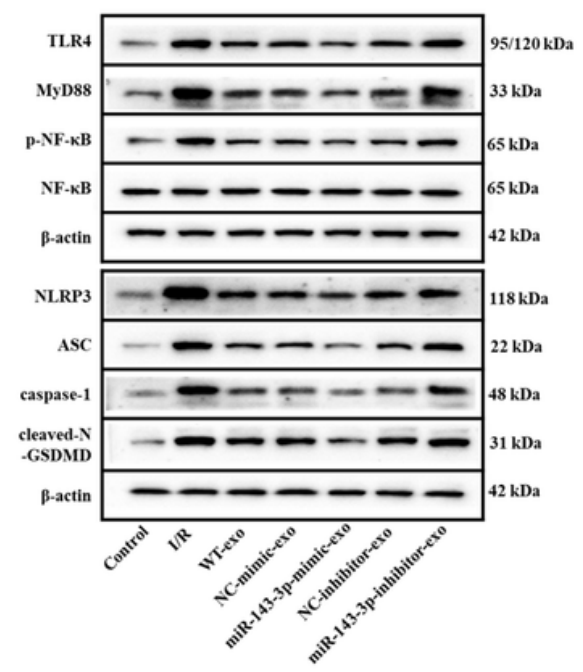

C
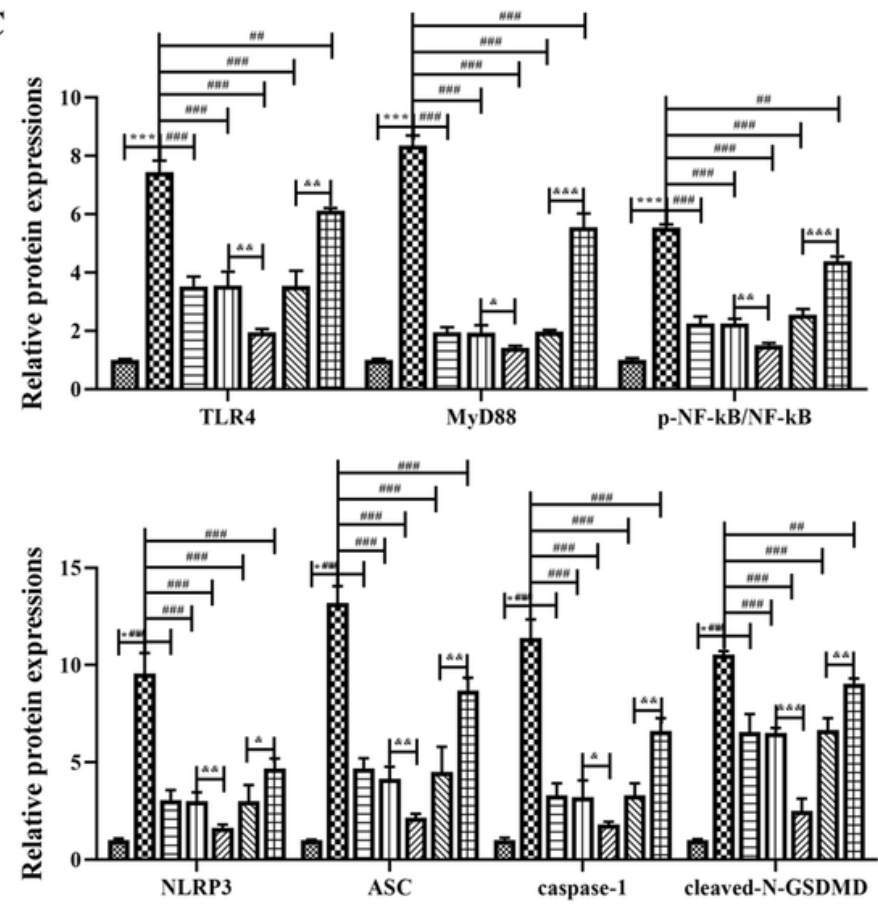

Control

$\mathbf{I} \mathbf{I} / \mathbf{R}$

口 WT-exo

四 NC minic-exo

miR-143-3p minic-exo

NC inhibitor-exo

miR-143-3p inhibitor-exo

Figure 9 
Effects of miR-143-3p in BMSC-exo on pyroptosis pathway in I/R model mice. (A) The expressions of IL1ß, IL-18 and TNF-a were detected by ELISA. (B) Protein expressions of TLR4, MyD88, p-NF-KB, NF-KB, NLRP3, ASC, caspase-1 and cleaved-N-GSDMD were detected by western blot analysis. (C) Densitometry analysis of the protein expression. Data were shown as mean $\pm \mathrm{SD} .{ }^{*} \mathrm{P}<0.05,{ }^{* \star} \mathrm{P}<0.01$ and ${ }^{\star \star *} \mathrm{P}<$ 0.001 , compared with the control group; ${ }^{\#} \mathrm{P}<0.05$, ${ }^{\# \#} \mathrm{P}<0.01$ and ${ }^{\# \# \#} \mathrm{P}<0.001$, compared with the $\mathrm{I} / \mathrm{R}$ model group; ${ }^{2} P<0.05$, \&\& $P<0.01$ and ${ }^{\& \& \&} P<0.001$, compared with the NC mimic-exo or NC inhibitorexo group. 